\title{
Measurements of the thermal dissipation field in turbulent Rayleigh-Bénard convection
}

\author{
Xiaozhou He and Penger Tong* \\ Department of Physics, Hong Kong University of Science and Technology, Clear Water Bay, Kowloon, Hong Kong
}

(Received 13 October 2008; published 6 February 2009)

\begin{abstract}
A systematic study of the thermal dissipation field and its statistical properties is carried out in turbulent Rayleigh-Bénard convection. A local temperature gradient probe consisting of four identical thermistors is made to measure the normalized thermal dissipation rate $\epsilon_{N}(\mathbf{r})$ in two convection cells filled with water. The measurements are conducted over varying Rayleigh numbers $\mathrm{Ra}\left(8.9 \times 10^{8} \leqq \mathrm{Ra} \lesssim 9.3 \times 10^{9}\right)$ and spatial positions $\mathbf{r}$ across the entire cell. It is found that $\epsilon_{N}(\mathbf{r})$ contains two contributions; one is generated by thermal plumes, present mainly in the plume-dominated bulk region, and decreases with increasing Ra. The other contribution comes from the mean temperature gradient, being concentrated in the thermal boundary layers, and increases with $\mathrm{Ra}$. The experiment provides a complete physical picture about the thermal dissipation field and its statistical properties in turbulent convection.
\end{abstract}

DOI: 10.1103/PhysRevE.79.026306

PACS number(s): 44.25.+f, 47.27.T-

\section{INTRODUCTION}

Fluid turbulence is often considered as a cascade process with turbulent kinetic energy being continuously transferred from the largest eddies of size $\ell_{0}$ to eddies of smaller size, until it dissipates when the size of the eddies becomes comparable to the viscous dissipation length $\ell_{d}$. In the inertial range $\ell_{d}<\ell<\ell_{0}$, the energy cascades at a constant rate $\epsilon_{u}$ without dissipation [1]. The Kolmogorov dissipation length $\ell_{d}$ is defined by equating the Reynolds number $\operatorname{Re}\left(\ell_{d}\right)$ $=\ell_{d} u\left(\ell_{d}\right) / \nu$ to unity, where $\nu$ is the kinematic viscosity of the fluid and $u(\ell)$ is the velocity difference associated with eddies of size $\ell$. Direct measurement of $\epsilon_{u}$ in fully developed turbulence is difficult, because it involves simultaneous measurements of nine components of the velocity gradient tensor with fine spatial resolution below $\ell_{d}$ [2]. Good temporal resolution is also required in order to follow the rapid development of intensive turbulent events.

In an analogous manner, the transfer of fluctuations of a passive scalar $\theta$, such as temperature or concentration of a contaminant, to smaller scales is described by the dissipation rate $\epsilon_{\theta}$, which measures the mixing rate at which fluctuations of $\theta$ (or $\theta^{2}$ ) are destroyed. Measurements of the local dissipation rate $\epsilon_{\theta}$ have been carried out in turbulent wind tunnels [3] and water jets [4]. These experiments aimed at studying the intermittent nature of passive scalar fluctuations in turbulent flows [5]. For a temperature field $T(\mathbf{r}, t)$, the thermal dissipation field is defined as

$$
\epsilon_{T}(\mathbf{r}, t)=\kappa|\nabla T(\mathbf{r}, t)|^{2},
$$

where $\kappa$ is the thermal diffusivity and $\nabla T(\mathbf{r}, t)$ is the temperature gradient field. The value of $\epsilon_{T}(\mathbf{r}, t)$ is always positive and the determination of $\epsilon_{T}(\mathbf{r}, t)$ involves simultaneous measurements of the three components of $\nabla T(\mathbf{r}, t)$.

In turbulent Rayleigh-Bénard convection, where a fluid layer of thickness $H$ is heated from below and cooled from the top, temperature becomes an active scalar which drives the convective turbulence. In this case, the thermal dissipa-

\footnotetext{
*penger@ust.hk
}

tion field $\epsilon_{T}(\mathbf{r}, t)$ is determined by the dynamics of the flow and the global average of $\epsilon_{T}(\mathbf{r}, t)$ becomes proportional to the total heat flux transported vertically through the convection cell. In particular, one finds [6]

$$
\left\langle\epsilon_{N}(\mathbf{r}, t)\right\rangle_{V, t} \equiv \frac{\left\langle\epsilon_{T}(\mathbf{r}, t)\right\rangle_{V, t}}{\kappa(\Delta T / H)^{2}}=\mathrm{Nu}(\operatorname{Ra}, \operatorname{Pr}),
$$

where $\epsilon_{N}(\mathbf{r}, t) \equiv \epsilon_{T}(\mathbf{r}, t) /\left[\kappa(\Delta T / H)^{2}\right]$ is the normalized thermal dissipation field, $\mathrm{Nu}(\mathrm{Ra}, \mathrm{Pr}$ ) is the Nusselt number (normalized heat flux), $\Delta T$ is the temperature difference across the cell of height $H$, and $\langle\cdots\rangle_{V, t}$ represents averages over the fluid volume $V$ and time $t$. There are two experimental control parameters in turbulent Rayleigh-Bénard convection. One is the Rayleigh number $\mathrm{Ra}=\alpha g \Delta T H^{3} /(\nu \kappa)$, where $g$ is the gravitational acceleration, and $\alpha, \nu$, and $\kappa$ are, respectively, the thermal expansion coefficient, the kinematic viscosity, and the thermal diffusivity of the convecting fluid. The other control parameter is the Prandtl number $\operatorname{Pr}=\nu / \kappa$.

The theory by Grossmann and Lohse (GL) [7-10] explains the scaling behavior of $\mathrm{Nu}(\mathrm{Ra}, \mathrm{Pr})$ by a decomposition of the dissipation field $\epsilon_{N}(\mathbf{r})=\left\langle\epsilon_{N}(\mathbf{r}, t)\right\rangle_{t}$ into two parts. In one scenario [7-9], $\epsilon_{N}(\mathbf{r})$ is decomposed into the boundary layer and bulk contributions, which have different scaling behavior with varying $\mathrm{Ra}$ and $\mathrm{Pr}$. More recently, a second scenario was proposed [10] with $\epsilon_{N}(\mathbf{r})$ being decomposed into two different contributions: Thermal plumes (including the boundary layers) and turbulent background. While the two scenarios involve different physical pictures about the local dynamics of turbulent convection, the calculated $\mathrm{Nu}(\mathrm{Ra}, \mathrm{Pr})$ using the two different models turns out to be of the same scaling form. This suggests that while the GL theory is capable of providing a correct functional form of $\mathrm{Nu}(\mathrm{Ra}, \mathrm{Pr})$ for a large number of transport measurements [11-19], the microscopic mechanism of heat transport and its connection to the local dynamics of turbulent convection still remain illusive.

In this paper, we report a systematic study of the dissipation field $\epsilon_{N}(\mathbf{r}, t)$ over varying Rayleigh numbers $\mathrm{Ra}$ and spatial positions $\mathbf{r}$ across the entire convection cell. It is found that $\epsilon_{N}(\mathbf{r})$ contains two contributions; one is generated 
by thermal plumes, present mainly in the plume-dominated bulk region, and decreases with increasing Ra. The other contribution comes from the mean temperature gradient, being concentrated in the thermal boundary layers, and increases with $\mathrm{Ra}$. The experiment provides a complete physical picture about the thermal dissipation field and its statistical properties in turbulent convection.

The remainder of the paper is organized as follows. We first describe the apparatus and the experimental method in Sec. II. Experimental results are discussed in Sec. III. Finally, the work is summarized in Sec. IV.

\section{EXPERIMENT}

The experiment is conducted in two upright cylindrical cells filled with water. The two convection cells have the same inner diameter of $D=19.0 \mathrm{~cm}$ but their height is different; one is $20.5 \mathrm{~cm}$ and the other is $40.9 \mathrm{~cm}$. The corresponding aspect ratio $(\Gamma=D / H)$ of the two cells is $\Gamma \simeq 1$ and $\Gamma \simeq 0.5$, respectively. Details about the apparatus and temperature measurements have been described elsewhere [20-22], and here we mention only some key points. The sidewall of the cells is made of a transparent Plexiglas ring with a wall thickness of $0.6 \mathrm{~cm}$. The top and bottom plates are made of brass and their surfaces are electroplated with a thin layer of gold. The thickness of the top plate is $1.0 \mathrm{~cm}$ and that of the bottom plate is $0.85 \mathrm{~cm}$. The Plexiglas ring is sandwiched between the two plates and is sealed to the top and bottom plates via two rubber $\mathrm{O}$ rings. Two silicon rubber film heaters connected in parallel are sandwiched on the backside of the bottom plate to provide constant and uniform heating. A dc power supply with $99.99 \%$ long-term stability is used to provide the heating power. The voltage applied to the heaters varies from 20 to 80 volts, and the corresponding heating power is in the range between $23 \mathrm{~W}$ and $371 \mathrm{~W}$. The upper side of the top plate together with a circular aluminum cover form a closed cooling chamber, whose temperature is maintained constant by circulating cold water from a temperature-controlled bath and/or circulator. The temperature stability of the circulator is $0.01{ }^{\circ} \mathrm{C}$.

The two cells are used to change the aspect ratio and to extend the accessible range of the Rayleigh number Ra. The $\Gamma=1$ cell covers the Ra range between $8.9 \times 10^{8}$ and 9.3 $\times 10^{9}$. In the $\Gamma=0.5$ cell, the maximum value of $\mathrm{Ra}$ can reach up to $5.9 \times 10^{10}$. The temperature difference $\Delta T$ between the top and bottom plates is measured by two thermistors embedded in each plate. In the experiment, the value of $\Delta T$ varies from $4.8^{\circ} \mathrm{C}$ to $50{ }^{\circ} \mathrm{C}$ depending on the heating power. By adjusting the temperature of the cooling water, we maintain the temperature of the bulk fluid at $\sim 30{ }^{\circ} \mathrm{C}$ for all the measurements. At this temperature, one has $\nu \simeq 8.2$ $\times 10^{-3} \mathrm{~cm}^{2} / \mathrm{s}, \kappa \simeq 1.5 \times 10^{-3} \mathrm{~cm}^{2} / \mathrm{s}$ and the corresponding Prandtl number, $\operatorname{Pr}=\nu / \kappa \simeq 5.5$. The temperature stability of the top and bottom plates is found to be within $0.1^{\circ} \mathrm{C}$ in standard deviation, which is less than $2 \%$ of the minimum $\Delta T$ used in the experiment. The convection cell is placed inside a closed square box, whose temperature is maintained at $30 \pm 0.3{ }^{\circ} \mathrm{C}$ to prevent heat exchange between the convecting fluid (water) and the surroundings.
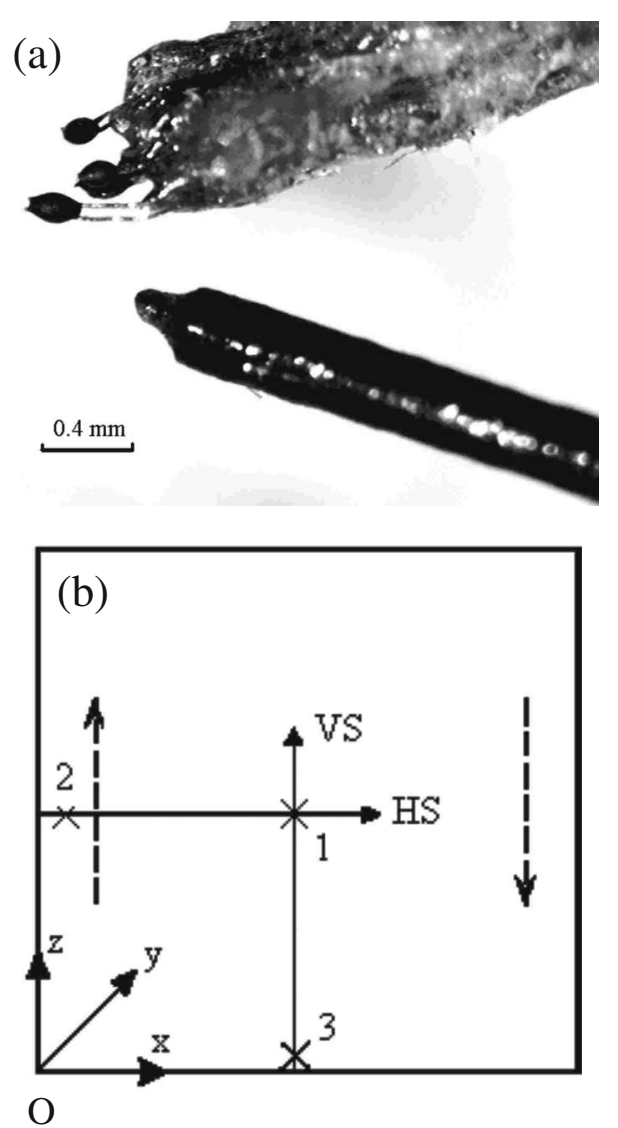

FIG. 1. (a) Upper figure shows the assembly of a homemade temperature gradient probe consisting of four identical thermistor beads. Lower figure shows a preassembled thermistor (AB6E3B05KA202R) purchased from GE Thermometrics. (b) Space coordinates used for the presentation of the measurements. The long horizontal (HS) and vertical (VS) arrows indicate, respectively, the tracks of the horizontal and vertical scans of the local dissipation measurements. The three crosses marked with a number indicate the positions used for the statistical study of dissipation fluctuations. The dashed arrows near the sidewall indicate the direction of the large-scale circulation.

Four identical thermistors are used to measure the three components of the local temperature gradient simultaneously. One of the thermistors is placed at the origin, labeled as $T_{0}$, and the other three thermistors are arranged along the $x, y$, and $z$ axis, respectively. By simultaneously measuring the four temperature signals, we obtain the three temperature gradient components $\delta T_{i} / \delta \ell$, where $\delta T_{i}=T_{i}$ $-T_{0}(i=x, y, z)$ is the temperature difference between a pair of the thermistors with separation $\delta \ell$. Two temperature gradient probes are used in the experiment. One consists of four preassembled thermistors with $0.17 \mathrm{~mm}$ in diameter and $\delta \ell$ $=0.8 \pm 0.1 \mathrm{~mm}$. The preassembled thermistors $(\mathrm{AB} 6 \mathrm{E} 3-$ B05KA202R) were purchased from GE Thermometrics and their construction is shown in the lower figure of Fig. 1(a). This probe is used to measure the spatial distribution of $\epsilon_{N}(\mathbf{r})$ at fixed values of $\mathrm{Ra}$ and its spatial resolution $\delta \ell$ is comparable to the thermal boundary layer thickness $\delta(\simeq 0.8 \mathrm{~mm}$ at $\mathrm{Ra}=3.6 \times 10^{9}$ ), which is the smallest length scale in turbulent convection. 
The second probe is made of four smaller thermistor beads of $0.11 \mathrm{~mm}$ in diameter and is assembled in our own laboratory, as shown in the upper figure of Fig. 1(a). The small thermistor beads (BB05JA243N) were purchased from GE Thermometrics. Each of them has a semiconductor head of $80 \mu \mathrm{m}$ in diameter and two 1-cm-long metal legs of $10 \mu \mathrm{m}$ in diameter. Silver paste is used to glue each of the metal legs to a $100-\mu$ m-diameter copper extension wire. The thermistor assembly is then coated with a thin layer of waterproof varnish for use in water. The second probe has a higher spatial resolution of $\delta \ell=0.25 \pm 0.1 \mathrm{~mm}$. This probe is used to measure the Ra dependence of $\epsilon_{N}(\mathbf{r})$ and the spatial distribution of $\epsilon_{N}(\mathbf{r})$ across the lower thermal boundary layer (at a fixed value of $\mathrm{Ra}$ ). All the thermistors are calibrated individually with an accuracy of $\sim 5 \mathrm{mK}$ for $\delta T_{i}$.

Each of the thermistors is connected to an ac transformer bridge as a resistor arm and the other resistor arm is connected to a variable resistor to balance the bridge. The bridge is driven by a lock-in amplifier (SR830, Stanford Research System) at a working frequency $f_{0} \simeq 1 \pm 0.2 \mathrm{kHz}$. Four identical bridges and lock-in amplifiers are used; each operates at a slightly different reference frequency (shifted by $100 \mathrm{~Hz}$ ) to avoid cross-talks between the four signals. The output signals are digitized simultaneously by a multichannel A-to-D I/O board (BNC2110, National Instrument) and the final data are stored in a host computer. The sampling rate of the temperature measurements is set at $40 \mathrm{~Hz}$. Typically, we take 2-h-long time series data $\left(2.88 \times 10^{5}\right.$ data points $)$ at each location for the measurement of the spatial distribution of $\epsilon_{N}(\mathbf{r})$ and 8 -h-long time series data $\left(1.15 \times 10^{6}\right.$ data points) at a fixed location for the study of the Ra dependence and the statistical properties of $\epsilon_{N}(\mathbf{r})$.

To guide the temperature gradient probe into the cell, we install a horizontal stainless steel tube on the sidewall. The stainless steel tube (type 304 SS hyperdermic tubing, $19 \mathrm{Ga}$, McMASTER-CARR) has an outer diameter $1.1 \mathrm{~mm}$ and wall thickness $0.19 \mathrm{~mm}$. Thin thermistor wires thread through the tube from the outside and a small head piece of the probe sticks out of the tube end inside the convection cell. The tube end is sealed with glue so that the convecting fluid cannot leak out through the stainless steel tube. The tube can slide in and out so that $\epsilon_{N}(\mathbf{r})$ can be measured at various positions. For the horizontal scan [see Fig. 1(b)], the tube is installed at the mid-height of the cell along a cell diameter. For the vertical scan, the tube is installed vertically through the center of the top plate so that $\epsilon_{N}(\mathbf{r})$ can be measured along the central axis of the cell.

Figure 1(b) shows the space coordinates to be used below in the presentation of the measurements of $\epsilon_{N}(\mathbf{r})$. The origin of the coordinate system is chosen to coincide with the lower left corner of the vertical cross section of the cell. The $x$ and $z$ axes are in the rotation plane of the large-scale circulation (LSC) and the $y$ axis is perpendicular to the rotation plane. The long horizontal (HS) and vertical (VS) arrows indicate, respectively, the tracks of the horizontal and vertical scans of the local dissipation measurements. The three crosses marked with a number indicate the positions used for the statistical study of fluctuations of the local thermal dissipation rate. To pin down the azimuthal motion of the LSC, we tip the cell with a small angle $\left(<1^{\circ}\right)$ by adding a few sheets

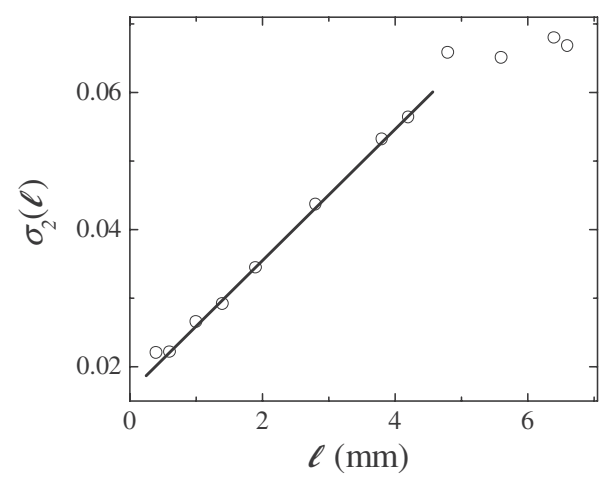

FIG. 2. Measured standard deviation $\sigma_{2}(\ell)$ as a function of separation $\ell$. The solid line shows a linear fit to the data point with small values of $\ell$. The measurements are made at the cell center with $\mathrm{Ra}=3.9 \times 10^{9}$.

of paper at position $O$ shown in Fig. 1(b). It has been shown [23] that such a small tilt does not affect the convective flow very much. When the cell is tilted at position $O$, the LSC is set up in the $x-z$ plane and the direction of the LSC is shown by the two long-dashed arrows in Fig. 1(b). All the measurements reported here are conducted in the rotation plane of the LSC.

\section{RESULTS AND DISCUSSION}

\section{A. Measurement of the local thermal dissipation rate}

To measure the local gradient of the temperature field, one needs to keep the separation $\delta \ell$ between the thermistors as small as possible. This separation should be smaller than the thermal boundary layer thickness $\delta$, which is the smallest dissipation length in turbulent thermal convection. At length scales smaller than $\delta$, temperature fluctuations are dissipated by diffusion. The value of $\delta$ decreases with increasing $\mathrm{Ra}$ $\left(\delta=425 \mathrm{Ra}^{-0.285} \mathrm{~mm}\right.$ [26]). On the other hand, the probe separation should be large enough to minimize the disturbances produced by a thermistor tip to the nearby temperature measurements. In the experiment, we set the thermistor's separation at a minimal value of $\delta \ell=0.8 \pm 0.1 \mathrm{~mm}$ for the first temperature gradient probe and $\delta \ell$ $=0.25 \pm 0.1 \mathrm{~mm}$ for the second probe. These values are $3-5$ times larger than the tip diameter of the thermistor but 1-3 times smaller than the measured value of $\delta$ at $\mathrm{Ra}=3.6$ $\times 10^{9}(\simeq 0.8 \mathrm{~mm})$.

Another way to examine whether the thermistor's separation $\delta \ell$ is set properly in the experiment is to directly measure the standard deviation of the temperature difference, $\sigma_{2}(\ell)=\left\langle\left[T_{2}(r+\ell)-T_{1}(r)\right]^{2}\right\rangle^{1 / 2}$, between two thermistors with varying separation $\ell$. Figure 2 shows the measured $\sigma_{2}(\ell)$ as a function of separation $\ell$. It is found that the measured $\sigma_{2}(\ell)$ is a linear function of $\ell$ for small values of $\ell$, with the ratio $\sigma_{2}(\ell) / \ell$ remaining constant in the range $\ell \leqq 4 \mathrm{~mm}$. Figure 2 thus suggests that the measured $\epsilon_{i}(\mathbf{r})=\kappa\left\langle\left(\delta T_{i} / \delta \ell\right)^{2}\right\rangle$ $\simeq \kappa\left[\sigma_{2}(\ell) / \ell\right]^{2}$ will remain constant, so long as that the thermistor's separation $\delta \ell$ chosen is in the linear region with $\ell$ $\$ 4 \mathrm{~mm}$. Here $\epsilon_{i}(\mathbf{r})$ is a component of $\epsilon_{T}(\mathbf{r})$. The thermistor's separation $\delta \ell$ chosen for the two temperature gradient probes are indeed in this linear region. 

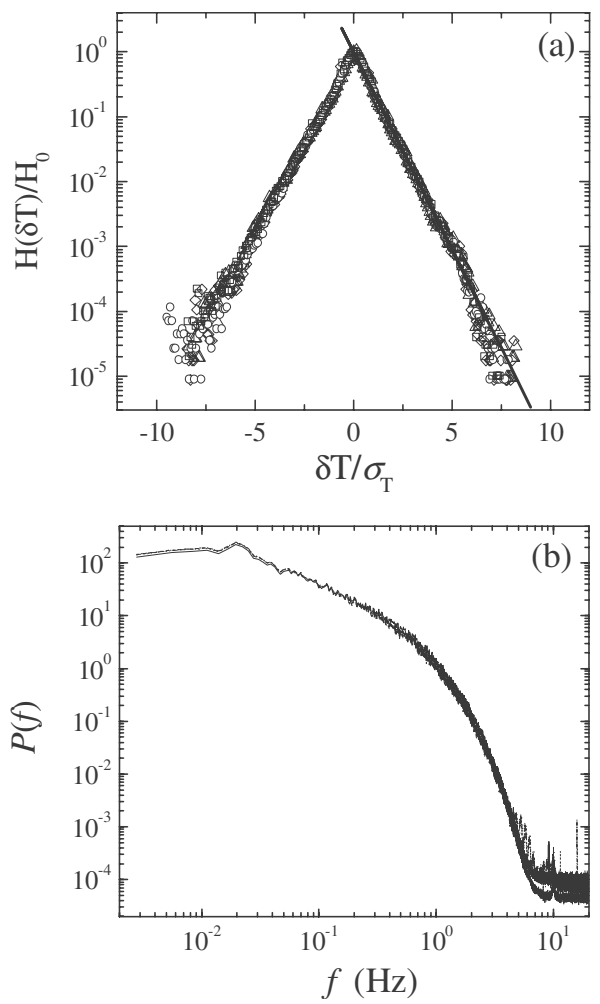

FIG. 3. (a) Measured temperature histograms $H(\delta T) / H_{0}$ as a function of $\delta T / \sigma_{T}$. Four histograms (circles, squares, triangles, and diamonds) are obtained using the four thermistors, of which the temperature gradient probe is made. The solid line shows the fitted function, $H(\delta T) / H_{0}=\exp \left[-\alpha\left(\delta T / \sigma_{T}\right)\right]$, with $\alpha=1.4$. (b) Frequency power spectra $P(f)$ of the temperature signals obtained from the four thermistors. All the measurements in (a) and (b) are made at the center of the $\Gamma \simeq 1$ cell with $\mathrm{Ra}=3.96 \times 10^{9}$.

Another issue in the measurement of $\epsilon_{N}(\mathbf{r})$ concerns with the perturbations to the local temperature measurement produced by the three nearby thermistors. To examine this effect, we compare the histograms $H(\delta T)$ of the local temperature signals measured by the four thermistors, of which the temperature gradient probe is made. Here the temperature fluctuation $\delta T$ is defined as $\delta T=T(\mathbf{r}, t)-\langle T\rangle$, with $\langle T\rangle$ being the local mean value of $T(\mathbf{r}, t)$. Figure $3(\mathrm{a})$ shows the resulting $H(\delta T) / H_{0}$ using the 8-h-long time series data obtained at $\mathrm{Ra}=3.96 \times 10^{9}$. In the plot, the measured $H(\delta T)$ is normalized by its peak value $H_{0}$, and the horizontal variable $\delta T$ is normalized by its standard deviation $\sigma_{T}=\langle[T(\mathbf{r}, t)$ $\left.-\langle T\rangle(\mathbf{r})]^{2}\right\rangle^{1 / 2}$. The histograms obtained from the four different thermistors superpose nicely, and they all show a simple exponential form (solid line) over an amplitude range of more than 5 decades. Such an exponential form was also observed previously in a similar convection system using a single thermistor probe [24]. Figure 3(b) shows the measured frequency power spectra $P(f)$ of the temperature signals obtained from the four thermistors. The four temperature power spectra superpose nicely, indicating that the temperature signal measured by one of the thermistors is not affected by the surrounding thermistors, at least in the statistical sense. Figure 3 thus demonstrates that the four thermistors at the chosen separation do not interfere with each other very much, so
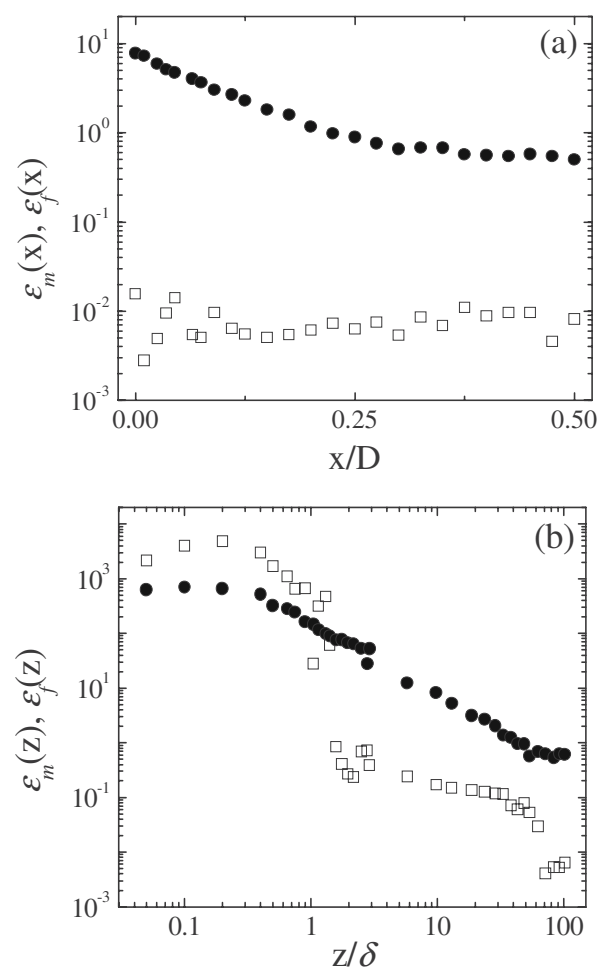

FIG. 4. (a) Comparison between $\epsilon_{m}(x)$ (open squares) and $\epsilon_{f}(x)$ (solid circles) obtained from the horizontal scan along a cell diameter at the mid-height of the cell. (b) Comparison between $\epsilon_{m}(z)$ (open squares) and $\epsilon_{f}(z)$ (solid circles) obtained from the vertical scan along the central axis of the cell. All the measurements in (a) and (b) are made in the $\Gamma \simeq 1$ cell with $\mathrm{Ra}=3.9 \times 10^{9}$.

that the measured temperature signals have the same statistical properties.

\section{B. Spatial distribution of the thermal dissipation field}

$$
\text { 1. } \Gamma \simeq 1 \text { cell }
$$

We first discuss the measurements of $\epsilon_{N}(\mathbf{r})$ in the $\Gamma=1$ cell. Some of the results described here have been reported briefly [25]. From Eq. (1), one finds that $\epsilon_{N}(\mathbf{r})$ contains three contributions, $\epsilon_{N}^{i}(\mathbf{r})(i=x, y, z)$, resulting from the three components of the temperature gradient. Each contribution can be further decomposed into two terms: $\epsilon_{N}^{i}(\mathbf{r})=\epsilon_{m}^{i}(\mathbf{r})+\epsilon_{f}^{i}(\mathbf{r})$, with $\epsilon_{m}^{i}(\mathbf{r})$ resulting from the mean temperature gradient and $\epsilon_{f}^{i}(\mathbf{r})$ coming from the fluctuations. As a result, the total dissipation can be written as $\epsilon_{N}(\mathbf{r})=\epsilon_{m}(\mathbf{r})+\epsilon_{f}(\mathbf{r})$. In the horizontal scan (HS) shown in Fig. 1(b), we measure $\epsilon_{N}(\mathbf{r})$ from one side of the cell to the other side along a cell diameter at the mid-height of the cell. In the vertical scan (VS), we measure $\epsilon_{N}(\mathbf{r})$ along the central axis of the cell from the center of the bottom plate to the center of the cell. All the measurements are conducted in the plane of the LSC.

Figure 4(a) compares the measured dissipation profiles $\epsilon_{m}(x)$ (open squares) and $\epsilon_{f}(x)$ (solid circles) as a function of the normalized horizontal position $x / D(x / D=0.5$ at the cell center). The measured $\epsilon_{m}(x)$ is negligibly small when compared with $\epsilon_{f}(x)$. We find that $\epsilon_{N}(\mathbf{r}) \simeq \epsilon_{f}(\mathbf{r})$ is true for all the measurements in the bulk region outside the thermal bound- 


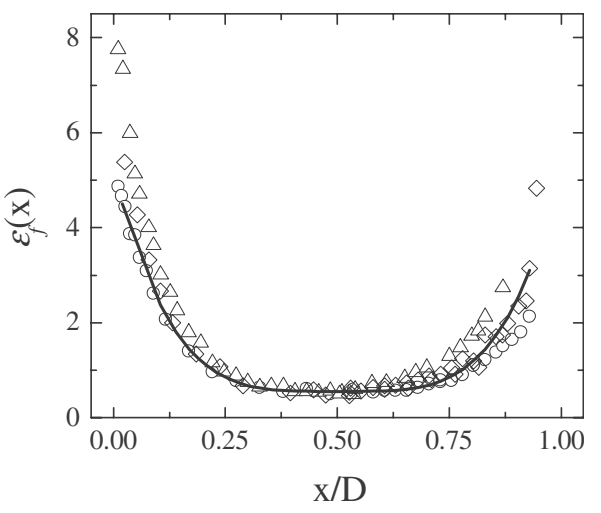

FIG. 5. Measured horizontal profile $\epsilon_{f}(x)$ as a function of $x / D$ for three different values of Ra: $6.6 \times 10^{9}$ (circles), $2.7 \times 10^{9}$ (triangles), and $9.2 \times 10^{8}$ (diamonds). The solid line shows the fitted function, $\epsilon_{f}(x)=a+b(x / D-0.5)^{4}$, to the circles with $a=0.56$ and $b=74.4$.

ary layer. Figure 4(b) compares $\epsilon_{m}(z)$ (open squares) with $\epsilon_{f}(z)$ (solid circles) obtained from the vertical scan along the central axis of the cell. The measured $\epsilon_{m}(z)$ shows drastic changes in three different regions of $z / \delta$. (i) In the $z / \delta \leqslant 1$ region (inside the thermal boundary layer), $\epsilon_{m}(z)$ is larger than $\epsilon_{f}(z)$ and the difference between the two quantities peaks at $z / \delta \simeq 0.2$. The largest value of the ratio $\epsilon_{m}(\mathbf{r}) / \epsilon_{f}(\mathbf{r})$ is 7.4 at $z / \delta \simeq 0.2$. The decrease of the measured $\epsilon_{m}(z)$ at smaller values of $z / \delta$ is an artifact due to the fact that the temperature gradient probe used has a finite spatial resolution of $\delta \ell \simeq 0.25 \mathrm{~mm}$. (ii) In the $1 \leqq z / \delta \leqq 60$ region, the measured $\epsilon_{m}(z)$ decreases sharply by a factor of $\sim 10^{3}$, making it much smaller than $\epsilon_{f}(z)$. (iii) In the $z / \delta \geq 60$ region, the measured $\epsilon_{m}(z)$ is further reduced by a factor of more than 10 , so that $\epsilon_{f}(z)$ remains dominant over $\epsilon_{m}(z)$.

Figure 5 shows the measured horizontal profiles of $\epsilon_{f}(x)$ as a function of $x / D$ for three different values of Ra. Because of the cylindrical symmetry of the cell, the measured $\epsilon_{f}(x)$ is symmetric about the central axis with a minimum value at the cell center and increases sharply in the sidewall region, in which both the local velocity and local convective heat flux reach maximum [22,27-29]. The value of $\epsilon_{f}(x)$ near the sidewall is $\sim 10$ times larger than that at the cell center. The overall amplitude of the horizontal profile $\epsilon_{f}(x)$ decreases slightly with increasing $\mathrm{Ra}$, but its general shape remains unchanged in the Ra range studied. The solid curve in Fig. 5 shows the fitted function, $\epsilon_{f}(x)=a+b(x / D-0.5)^{4}$, to the circles with $a=0.56$ and $b=74.4$.

Figure 6(a) shows the measured vertical profiles of $\epsilon_{f}(z)$ as a function of the normalized vertical position $z / \delta$ for different values of Ra. The measured $\epsilon_{f}(z)$ has a minimal value at the cell center $(z / \delta \simeq 100)$ and reaches maximum near the lower conducting plate. When compared with Fig. 5, we find $\epsilon_{f}(z)$ increases more rapidly near the conducting plate. The maximal value of $\epsilon_{f}(z)$ at $z / \delta \simeq 1$ is $\sim 140$ times larger than the minimal value at the cell center. Another important feature shown in Fig. 6(a) is that the measured profiles $\epsilon_{f}(z)$ at different values of $\mathrm{Ra}$ superpose nicely, once the vertical position $z$ is normalized by the boundary layer thickness $\delta$.

Figure 6(b) shows the resulting vertical profiles of the total thermal dissipation rate $\epsilon_{N}(z)$ as a function of $z / \delta$ for

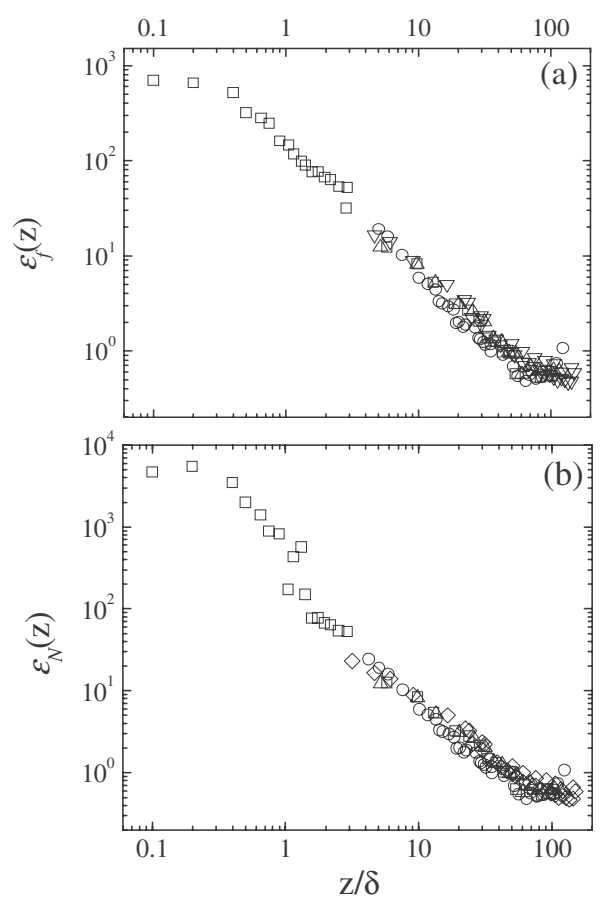

FIG. 6. (a) Measured vertical profile $\epsilon_{f}(z)$ as a function of $z / \delta$ for different values of Ra. (b) Measured vertical profile $\epsilon_{N}(z)$ as a function of $z / \delta$ for different values of Ra. The measurements in (a) and (b) are made in the $\Gamma \simeq 1$ cell with $\mathrm{Ra}=9.2 \times 10^{8}$ (triangles), $2.7 \times 10^{9}$ (diamonds), $3.9 \times 10^{9}$ (squares), and $6.6 \times 10^{9}$ (circles).

different values of Ra. As discussed earlier, in the bulk region of the cell we find $\epsilon_{N}(z) \simeq \epsilon_{f}(z)$, whereas near the boundary layer $\epsilon_{N}(z)$ is taken over by $\epsilon_{m}(z)$. Figure 6(b) clearly reveals that $\epsilon_{N}(z)$ is dominated by the boundary layer contribution. This is because the mean temperature difference $\Delta T / 2$ across the thermal boundary layer gives the largest temperature gradient [and hence the largest $\epsilon_{m}(z)$ ].

As mentioned earlier, the measured $\epsilon_{f}(\mathbf{r})$ contains three terms: $\epsilon_{f}^{i}(\mathbf{r})=\left(d T / d x_{i}\right)^{2} /(\Delta T / H)^{2} \quad(i=x, y, z)$. Figure 7(a) shows the measured horizontal profiles $\epsilon_{f}^{x}(x)$ (diamonds), $\epsilon_{f}^{y}(x)$ (circles), and $\epsilon_{f}^{z}(x)$ (triangles) as a function of $x / D$. As expected, the three horizontal profiles are symmetric about the central axis of the cell and their minimal value is located at the cell center. Because the flow in the central core region is nearly isotropic, the three terms of $\epsilon_{f}(\mathbf{r})$ have almost the same value. In the sidewall region, $\epsilon_{f}^{z}(x)$ becomes approximately 2 times larger than $\epsilon_{f}^{x}(x)$ and $\epsilon_{f}^{y}(x)$. The values of $\boldsymbol{\epsilon}_{f}^{x}(x)$ and $\boldsymbol{\epsilon}_{f}^{y}(x)$ are approximately the same because of the azimuthal symmetry of the flow system. By carefully comparing the time series data of the local temperature and local temperature gradient, we find that the large value of $\epsilon_{f}^{z}(x)$ comes from fluctuations of the temperature gradient produced by the rising (or falling) thermal plumes. Figure 7(b) shows the measured vertical profiles $\epsilon_{f}^{x}(z)$ (diamonds), $\epsilon_{f}^{y}(z)$ (circles), and $\epsilon_{f}^{z}(z)$ (triangles) as a function of $z / \delta$. Similar to the horizontal scan, the vertical scan also reveals that the dominant contribution to $\epsilon_{f}(z)$ is from $\epsilon_{f}^{z}(z)$. Near the edge of the thermal boundary layer $(z / \delta \gtrsim 1), \epsilon_{f}^{z}(z)$ is approximately 4 times larger than $\epsilon_{f}^{x}(x)$ and $\epsilon_{f}^{y}(z)$. 

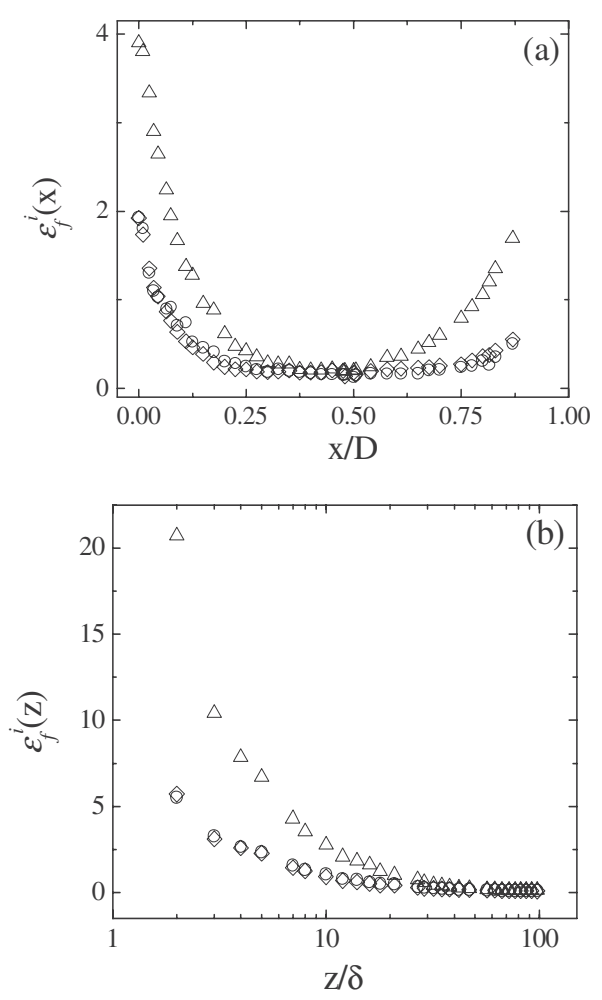

FIG. 7. (a) Measured horizontal profiles $\epsilon_{f}^{x}(x)$ (diamonds), $\epsilon_{f}^{y}(x)$ (circles), and $\epsilon_{f}^{z}(x)$ (triangles) as a function of $x / D$. (b) Measured vertical profiles $\epsilon_{f}^{x}(z)$ (diamonds), $\epsilon_{f}^{y}(z)$ (circles), and $\epsilon_{f}^{z}(z)$ (triangles) as a function of $z / \delta$. All the measurements in (a) and (b) are conducted in the $\Gamma \simeq 1$ cell at $\mathrm{Ra}=2.7 \times 10^{9}$.

$$
\text { 2. } \Gamma \simeq 0.5 \text { cell }
$$

We also measure the spatial distribution of $\epsilon_{f}(\mathbf{r})$ in the $\Gamma \simeq 0.5$ cell. Similar to the $\Gamma \simeq 1$ cell, the dominant contribution to $\epsilon_{N}(\mathbf{r})$ in the bulk region of the $\Gamma \simeq 0.5$ cell comes from the fluctuation term $\epsilon_{f}(\mathbf{r})$ and the mean gradient term $\boldsymbol{\epsilon}_{m}(\mathbf{r})$ is negligibly small. Figure 8(a) shows the measured horizontal profiles of $\epsilon_{f}(x)$ as a function of $x / D$ for two different values of Ra. The spatial distribution of $\epsilon_{f}(\mathbf{r})$ in the $\Gamma \simeq 0.5$ cell is similar to that in the $\Gamma \simeq 1$ cell. The measured $\epsilon_{f}(x)$ is symmetric about the central axis with a minimum value at the cell center and increases quickly in the sidewall region. Because the $\mathrm{LSC}$ in the $\Gamma \simeq 0.5$ cell is less stable compared with that in the $\Gamma \simeq 1$ cell (it wobbles even when the cell is tilted at a larger angle), the measured rms velocities are larger than their mean values in the entire region of the cell [27]. Consequently, the measured $\epsilon_{f}(x)$ at the cell center is larger than that in the $\Gamma \simeq 1$ cell. The overall amplitude of the horizontal profile $\epsilon_{f}(x)$ decreases with increasing $\mathrm{Ra}$, a trend which is also seen in the $\Gamma \simeq 1$ cell.

Figure $8(\mathrm{~b})$ shows the measured vertical profiles $\epsilon_{f}(z)$ as a function of $z$ / $\delta$ for two different values of Ra. Similar to the horizontal scan, the measured $\epsilon_{f}(z)$ has a minimal value at the cell center $(z / \delta \simeq 100)$ and increases sharply near the lower conducting plate. The maximal value of $\epsilon_{f}(z)$ at $z / \delta$ $\simeq 2$ is approximately 200 times larger than the minimal value at the cell center. As in the $\Gamma \simeq 1$ cell, the measured vertical profiles $\epsilon_{f}(z)$ at different values of Ra scale with the boundary layer thickness $\delta$.
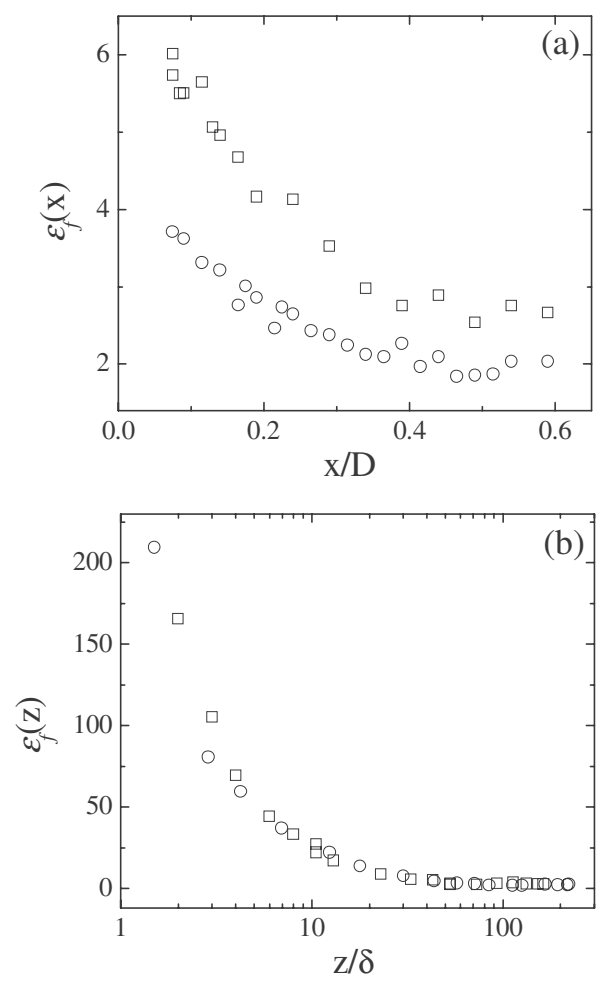

FIG. 8. (a) Measured horizontal profile $\epsilon_{f}(x)$ as a function of $x / D$ for two different values of Ra. (b) Measured vertical profile $\epsilon_{f}(z)$ as a function of $z / \delta$ for two different values of Ra. The measurements in (a) and (b) are made in the $\Gamma \simeq 0.5$ cell with $\mathrm{Ra}=9.1$ $\times 10^{9}$ (squares) and $2.7 \times 10^{10}$ (circles).

From the measurements in both the $\Gamma \simeq 1$ and $\Gamma \simeq 0.5$ cells, we arrive at the following physical picture about the thermal dissipation field in turbulent convection. The thermal dissipation field can be divided into two regions. (i) In the bulk region outside the thermal boundary layers, the dominant contribution to $\epsilon_{N}(\mathbf{r})$ comes from fluctuations of the local temperature gradient. The dissipation field $\epsilon_{f}(\mathbf{r})$ is generated by the detached thermal plumes and occupies mainly in the plume-dominated region near the sidewall and near the boundary layers $(z / \delta>1)$. In the central region of the cell, the measured $\epsilon_{f}(\mathbf{r})$ has a minimal value because of the small number of thermal plumes in the region. (ii) Inside the thermal boundary layers $(z / \delta \leqq 1)$, the dominant contribution to $\epsilon_{N}(\mathbf{r})$ comes from the mean temperature gradient in the direction perpendicular to the conducting surfaces. Such a spatial decomposition of $\epsilon_{N}(\mathbf{r})=\epsilon_{f}(\mathbf{r})+\epsilon_{m}(\mathbf{r})$ is consistent with the first scenario of the GL theory [7].

\section{Ra dependence of the local thermal dissipation rate}

Although the spatial distribution of $\epsilon_{N}(\mathbf{r})$ does not change very much in the range of Ra studied, the absolute value of $\epsilon_{N}(\mathbf{r})$ does change with Ra. We now discuss the Ra dependence of $\epsilon_{N}(\mathbf{r})$ at three representative locations in the convection cell. As indicated in Fig. 1(b), the three locations are marked as position 1 (at the cell center far away from the boundaries), position 2 (at the mid-height $2 \mathrm{~cm}$ away from the sidewall) and position 3 (at variable distances above the 


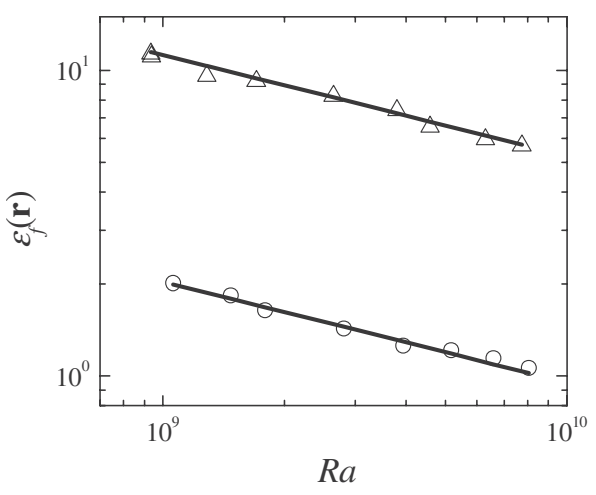

FIG. 9. Measured $\epsilon_{f}(\mathbf{r})$ as a function of Ra at the cell center (circles) and near the sidewall (triangles). The solid lines show the power-law fits, $\epsilon_{f}=\alpha \mathrm{Ra}^{\beta}$, with $\beta=0.33$ and $\alpha=1.9 \times 10^{3}$ (lower curve) and $\alpha=1.05 \times 10^{4}$ (upper curve).

center of the lower conducting surface). Figure 9 shows the measured $\epsilon_{f}(\mathbf{r})$ as a function of Ra at position 1 (circles) and position 2 (triangles). The measured $\epsilon_{f}(\mathbf{r})$ at the cell center and near the sidewall is well described by a power law $\epsilon_{f}(\mathbf{r})=\alpha \mathrm{Ra}^{-\beta}$ (solid lines) with the same value of $\beta$ $=0.33 \pm 0.03$ for both sets of the data. The power-law amplitude $\alpha$ for the circles is $1.9 \times 10^{3}$ and that for the triangles is $1.05 \times 10^{4}$. As shown in Fig. 4(a), the contribution of $\epsilon_{m}$ at the two positions is negligibly small.

Figure 10(a) shows the measured $\epsilon_{f}(\mathbf{r})$ as a function of Ra near the lower conducting plate at distance $\sim 1 \mathrm{~mm}$ above
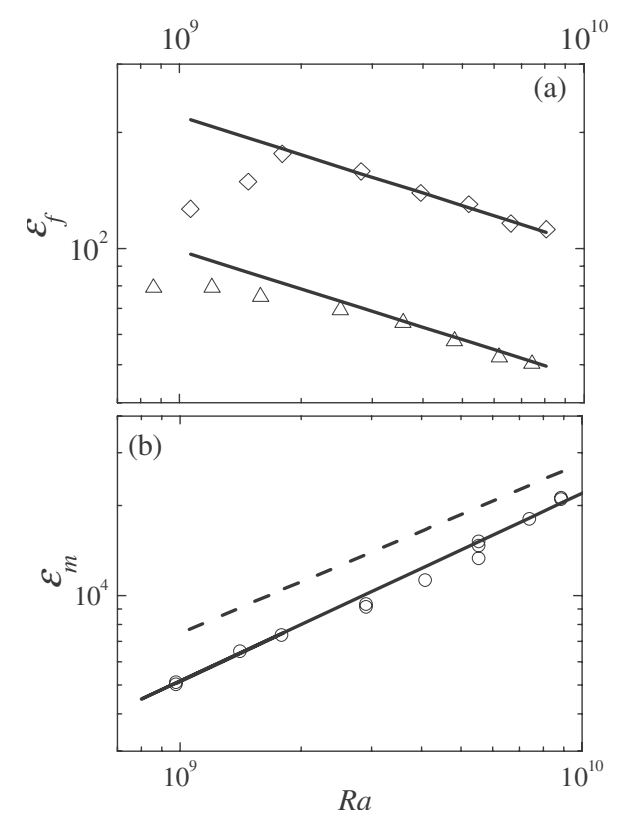

FIG. 10. (a) Measured $\epsilon_{f}(\mathbf{r})$ as a function of Ra near the lower conducting plate at distance $\sim 1 \mathrm{~mm}$ above the bottom plate (diamonds) and $\sim 2 \mathrm{~mm}$ above the bottom plate (triangles). The solid lines indicate the power-law fits, $\epsilon_{f}=\alpha \mathrm{Ra}^{\beta}$, with $\beta=0.33$ and $\alpha$ $=2.05 \times 10^{5}$ (upper curve) and $\alpha=9.2 \times 10^{4}$ (lower curve). (b) Measured Ra dependence of $\epsilon_{m}(\mathbf{r})$ inside the thermal boundary layer $(\sim 0.2 \mathrm{~mm}$ above the bottom plate). The solid line is a power-law fit, $\epsilon_{m}=1.1 \times 10^{-2} \mathrm{Ra}^{0.63}$. The dashed line shows $(H / 2 \delta)^{2} \simeq 5.54$ $\times 10^{-2} \mathrm{Ra}^{0.57}$ (see text). the bottom plate (diamonds) and $\sim 2 \mathrm{~mm}$ above the bottom plate (triangles). The measured $\boldsymbol{\epsilon}_{f}(\mathbf{r})$ reveals a crossover behavior. For example, the diamonds shown in Fig. 10(a) first increase with $\mathrm{Ra}$ for small values of Ra and then decrease at larger values of $\mathrm{Ra}$. This is because the measuring position at small values of $\mathrm{Ra}$ is located inside the thermal boundary layer. As the value of Ra increases, the boundary layer thickness decreases and the measuring position moves outside the boundary layer. The large-Ra portion of the data can be described by the same power law, $\epsilon_{f}=\alpha \mathrm{Ra}^{\beta}$, with $\beta=0.33$ and $\alpha=2.05 \times 10^{5}$ (upper curve) and $\alpha=9.2 \times 10^{4}$ (lower curve).

To obtain the Ra dependence of $\epsilon_{m}(\mathbf{r})$ inside the thermal boundary layer, we place the temperature gradient probe even closer to the lower conduction plate $(\sim 0.2 \mathrm{~mm}$ above the bottom plate) and the result is shown in Fig. 10(b). The solid line is a power-law fit, $\epsilon_{m}=1.1 \times 10^{-2} \mathrm{Ra}^{\gamma}$, with $\gamma$ $=0.63 \pm 0.05$. Because of finite heat capacity of the conducting plates and finite spatial resolution of the temperature gradient probe used, the obtained value of $\gamma$ has relatively larger uncertainties. However, the qualitative difference in the Ra dependence between $\epsilon_{f}(\mathbf{r})$ and $\epsilon_{m}(\mathbf{r})$ shown in Figs. 9 and 10 is so robust that it will not be affected by these experimental uncertainties.

While the spatial decomposition of $\epsilon_{N}(\mathbf{r})=\epsilon_{f}(\mathbf{r})+\epsilon_{m}(\mathbf{r})$ is consistent with the first scenario of the GL theory [7], the measured Ra dependence of $\epsilon_{f}(\mathbf{r})$ and $\epsilon_{m}(\mathbf{r})$ does not agree with this scenario. It is found that $\epsilon_{f}(\mathbf{r})$ throughout the bulk region scales as $\mathrm{Ra}^{-0.33}$, whereas $\epsilon_{m}(\mathbf{r})$ inside the thermal boundary layers scales with Ra differently $\left[\boldsymbol{\epsilon}_{m}(\mathbf{r}) \sim \mathrm{Ra}^{0.63}\right]$. The observed Ra dependence has two important implications. First, the volume average ratio $\left\langle\epsilon_{f}(\mathbf{r})\right\rangle_{V} /\left\langle\epsilon_{m}(\mathbf{r})\right\rangle_{V}$ will be a decreasing function of Ra, a trend which agrees with the recent numerical results $[30,31]$ but is opposite to that given by the first scenario of the GL theory $[7,10]$. Second, the measurements clearly reveal two competing effects of turbulence. On the one hand, temperature fluctuations are destroyed in the bulk region and their contributions to $\epsilon_{N}(\mathbf{r})$ decrease with increasing Ra. On the other hand, the temperature gradient (and hence the thermal dissipation) is enhanced near the conducting plates, because of the thinning of the boundary layers with increasing Ra. These two competing effects thus suggest that thermal plumes and the boundary layers are two different dynamic structures and cannot be treated equally, as suggested by the second scenario of the GL theory $[7,10]$.

Using the simple boundary layer scaling $[7,10]$, one finds that $\epsilon_{m}(\mathbf{r}) \simeq(H / 2 \delta)^{2}$, which is an upper bound for $\epsilon_{m}(\mathbf{r})$ without taking fluctuations into account. Therefore, we have $\left\langle\epsilon_{m}(\mathbf{r})\right\rangle_{V} \simeq(H / 2 \delta)^{2}(2 \delta / H) \simeq \mathrm{Nu}$, indicating that the boundary layers account for all the contributions to $\mathrm{Nu}$. The dashed line in Fig. 10(b) shows the measured $(H / 2 \delta)^{2} \simeq 5.54$ $\times 10^{-2} \mathrm{Ra}^{0.57}[26]$, which has a larger amplitude but smaller exponent when compared with the measured $\epsilon_{m}(\mathbf{r})$. The two curves intersect at $\mathrm{Ra}_{c} \simeq 1.68 \times 10^{11}$, above which one expects that $\epsilon_{N}(\mathbf{r})$ will be dominated by the boundary layer contributions. In fact, the recent heat transport measurements [14-19] have shown that $\mathrm{Nu} \sim \mathrm{Ra}^{1 / 3}$ for $\mathrm{Ra} \gtrsim \mathrm{Ra}_{c}$, which is a classical scaling based on a simple boundary-layer argument $[6,7]$. The measured Ra dependence of $\epsilon_{f}(\mathbf{r})$ and $\epsilon_{m}(\mathbf{r})$ thus provides a microscopic explanation to the crossover to the boundary-layer dominant state at $\mathrm{Ra} \gtrsim \mathrm{Ra}_{c}$. 
The above boundary-layer scaling arguments, however, neglect fluctuations of the thermal boundary layer due to the detachment of thermal plumes, which can cause a (momentary) increase of the boundary layer thickness (mostly occurred in the corner region of the convection cell $[26,27]$ ), resulting in a slight decrease of the thermal dissipation inside the boundary layers. The detached thermal plumes are then transported to and dissipated in the bulk region by the largescale flow. As an estimate, we calculate the spatially averaged dissipation

$$
\left\langle\epsilon_{f}(\mathbf{r})\right\rangle_{V}=\int_{V} \epsilon_{f}(\mathbf{r}) d \mathbf{r} \simeq \int\left[\epsilon_{f}(x) \epsilon_{f}(z) / \epsilon_{f}(0)\right] 2 \pi x d x d z .
$$

In obtaining the last equality, we have assumed that $\epsilon_{f}(\mathbf{r})$ is axially symmetric. Using the measured profiles of $\epsilon_{f}(x)$ and $\epsilon_{f}(z)$, we find $\left\langle\epsilon_{f}(\mathbf{r})\right\rangle_{V} \approx 8.1$ at $\mathrm{Ra}=2.7 \times 10^{9}$. This accounts for $\sim 9.7 \%$ of the measured $\mathrm{Nu}$ at this value of $\mathrm{Ra}$ [26]. Because $\epsilon_{f}(\mathbf{r})$ decreases with increasing Ra, the onset of the $\mathrm{Nu} \sim \mathrm{Ra}^{1 / 3}$ scaling at $\mathrm{Ra} \gtrsim \mathrm{Ra}_{c}$ may be viewed as a manifestation of diminishing contribution of $\epsilon_{f}(\mathbf{r})$ to the total dissipation rate.

\section{Fluctuations of the local thermal dissipation rate}

Large thermal fluctuations in turbulent convection are generated primarily by the thermal plumes emitted from the upper and lower thermal boundary layers. The role of thermal plumes played in terms of their spatial distribution, interactions with the mean flow, their signature in temperature time series data, and their effect on the local convective heat flux has been studied extensively in recent years $[20,24,27,29,32,33]$. The recent temperature, velocity and flow visualization measurements $[27,32,34,35]$ showed that the spatial distribution of thermal plumes in a closed cell is neither homogeneous nor isotropic. The thermal plumes organize themselves in such a way that warm plumes accumulate on one side of the cell and cold plumes concentrate on the opposite side of the cell. The spatially separated warm and cold plumes exert buoyancy forces on the fluid and drive the vertical flow near the sidewall. The central core region is "sheared" by the rising and falling plumes, resulting in a large-scale circulation (LSC) across the cell height. This large-scale circulation provides a fast channel along the cell periphery for the transport of heat $[22,29]$.

With this understanding of plume dynamics, we discuss in this section the statistical properties of the instantaneous thermal dissipation rate $\epsilon_{f}(\mathbf{r}, t)$ at the three representative locations in the convection cell. As indicated in Fig. 1(b), the three locations are all in the circulation plane of the LSC. Because the behavior of the dissipation fluctuations in the $\Gamma \simeq 0.5$ cell is similar to that in the $\Gamma \simeq 1$ cell, we focus our attention hereinafter to the measurements in the $\Gamma \simeq 1$ cell.

\section{At the cell center}

Figure 11 shows the time series data for the local temperature fluctuation $\delta T(t), z$ component of the temperature gradient $(d T / d z)(t)$, two components of the thermal dissipation rate $\epsilon_{f}^{x}(t)$ and $\epsilon_{f}^{z}(t)$, and the total dissipation date $\epsilon_{f}(t)$ (top to

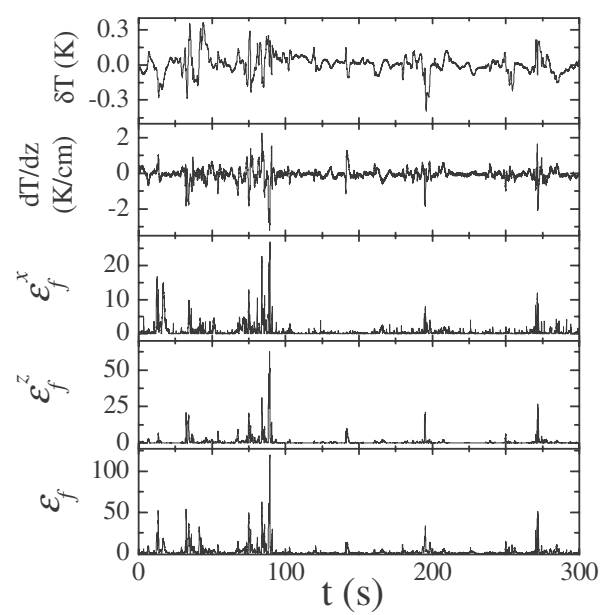

FIG. 11. Time series measurements of the local temperature fluctuation $\delta T(t), z$ component of the temperature gradient $(d T / d z)(t)$, two components of the thermal dissipation rate $\epsilon_{f}^{x}(t)$ and $\epsilon_{f}^{z}(t)$, and the total dissipation date $\epsilon_{f}(t)$ (top to bottom curves). All the measurements are made at the center of the $\Gamma \simeq 1$ cell with $\mathrm{Ra}=3.9 \times 10^{9}$.

bottom curves). In the central region of the cell, the warm and cold plumes are mixed up by the convective flow. Nevertheless, there are still large thermal plumes remaining in the region, producing temperature spikes of irregular sharp and variable heights. These temperature fluctuations are symmetric relative to the mean fluid temperature $T_{0}$. The measured temperature histogram $H(\delta T)$ has a simple (symmetric) exponential form [see Fig. 3(a)], which was also observed previously in other convection experiments $[11,24]$. The measured local temperate derivative $(d T / d z)(t)$ also shows spikes of irregular sharp and variable heights. A key signature of the plume-generated temperature fluctuations is their strong correlation with the resulting fluctuations in the temperature derivatives and the thermal dissipation rate. This is clearly shown in Fig. 11. It is seen that both the warm and cold plumes generate sharp spikes in $(d T / d z)(t)$.

Figure 12 shows the measured histograms $H(d T / d x) / H_{0}$ (diamonds), $H(d T / d y) / H_{0}$ (circles), and $H(d T / d z) / H_{0}$ (triangles) for the three components of the temperature gradient vector at the cell center. To display the three histograms in the same graph, we normalize the histograms by their maximum value $H_{0}$, and the gradient variables $d T / d x_{i} \quad\left(x_{i}\right.$ $=x, y, z)$ are normalized by their standard deviation $\sigma_{g}$. The mean value of the temperature gradient at the cell center is zero, and fluctuations of the two horizontal components are symmetric relative to the zero mean. Over an amplitude range of almost 5 decades, the measured $H(d T / d x) / H_{0}$ and $H(d T / d y) / H_{0}$ overlap very well and can be fit to a stretched exponential function,

$$
H\left(d T / d x_{i}\right)=H_{0} e^{-c\left[\left(d T / d x_{i}\right) / \sigma_{g}\right]^{d}},
$$

with $c=2.25$ and $d=0.64$ (solid curve). The measured $H(d T / d z) / H_{0}$ has approximately the same shape as $H(d T / d x) / H_{0}$ and $H(d T / d y) / H_{0}$, but a careful examination reveals that $H(d T / d z) / H_{0}$ is slightly asymmetric in the tail part of the distribution [when $H(d T / d z) / H_{0}<10^{-3}$ ]. 


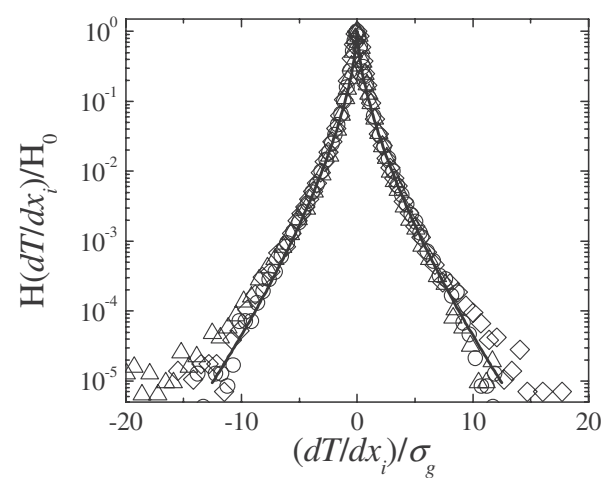

FIG. 12. Measured histograms $H(d T / d x) / H_{0}$ (diamonds), $H(d T / d y) / H_{0}$ (circles), and $H(d T / d z) / H_{0}$ (triangles) for the three components of the temperature gradient at the cell center with $\mathrm{Ra}$ $=3.96 \times 10^{9}$. The solid curve shows the fitted function, $H\left(d T / d x_{i}\right) / H_{0}=\exp \left\{-c\left[\left(d T / d x_{i}\right) / \sigma_{g}\right]^{d}\right\} \quad\left(x_{i}=x, y\right)$, to the diamonds and circles with $c=2.25$ and $d=0.64$.

Figure 13(a) shows the measured histograms $H\left(\epsilon_{f}\right)$ of the local dissipation rate $\epsilon_{f}$ at the cell center. The histograms obtained at different values of $\mathrm{Ra}$ can all be brought into coincidence, once $H\left(\epsilon_{f}\right)$ is normalized by its maximum value $H_{0}$ and $\epsilon_{f}$ is scaled by its rms value $\sigma_{\epsilon}$. Plots of $H\left(\epsilon_{f}\right) / H_{0}$ vs
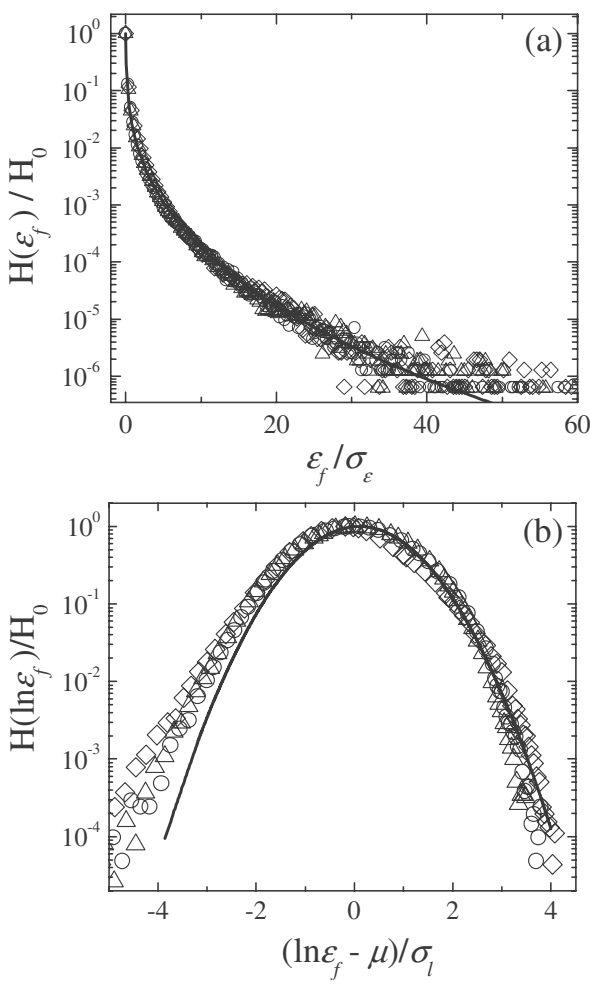

FIG. 13. (a) Measured histograms $H\left(\epsilon_{f}\right) / H_{0}$ as a function of $\epsilon_{f} / \sigma_{\epsilon}$. The measurements are made at the cell center with three values of $\mathrm{Ra}: 1.7 \times 10^{9}$ (diamonds), $3.9 \times 10^{9}$ (circles), and 8.2 $\times 10^{9}$ (triangles). The solid line shows the fitted function, $H\left(\epsilon_{f}\right) / H_{0}=\exp \left[-c\left(\epsilon_{f} / \sigma_{\epsilon}\right)^{d}\right]$, with $c=3.9$ and $d=0.35$. (b) Histograms $H\left[\ln \left(\epsilon_{f}\right)\right] / H_{0}$ as a function of the reduced variable, $k$ $\equiv\left[\ln \left(\epsilon_{f}\right)-\mu\right] / \sigma_{l}$, using the same data in (a). The solid line shows a Gaussian fit, $H(k) / H_{0}=\exp \left[-\left(k-\mu^{\prime}\right)^{2} / 2 \sigma_{l}^{\prime} 2\right]$, with $\mu^{\prime}=0.1$ and $\sigma_{l}^{\prime}=0.92$. $\epsilon_{f} / \sigma_{\epsilon}$ remain unchanged in the Ra range studied and only $\sigma_{\epsilon}$ changes with $\mathrm{Ra}$. The histograms have a universal form, which can be described by a stretched exponential function, $H\left(\epsilon_{f}\right) / H_{0}=\exp \left[-c\left(\epsilon_{f} / \sigma_{\epsilon}\right)^{d}\right]$, over an amplitude range of almost six decades. The solid curve shows the fit with $c=3.9$ and $d=0.35$. By carefully examining the time series data shown in Fig. 11, we find that the sharp rise of the measured $H\left(\epsilon_{f}\right) / H_{0}$ for small values of $\epsilon_{f} / \sigma_{\epsilon}$ are caused mainly by those small fluctuations near the baseline.

To display the probability density function of these smallamplitude fluctuations more clearly, we show, in Fig. 13(b), the histograms $H\left[\ln \left(\epsilon_{f}\right)\right]$ of $\ln \left(\epsilon_{f}\right)$ using the same data in Fig. $13(\mathrm{a})$. In the plot a reduced variable, $k \equiv\left[\ln \left(\epsilon_{f}\right)-\mu\right] / \sigma_{\ell}$, is used for the horizontal axis. Here $\mu$ is the mean value of $\ln \left(\epsilon_{f}\right)$ and $\sigma_{\ell}$ is its standard deviation; both are the averaged values over the entire data set. It is found that the values of $\mu$ and $\sigma_{\ell}$ change with Ra, but plots of $H\left[\ln \left(\epsilon_{f}\right)\right] / H_{0}$ vs $k$ remain unchanged in the Ra range studied. Two important features are observed from Fig. 13(b). (i) The measured $H\left[\ln \left(\epsilon_{f}\right)\right]$ for different values of Ra obeys the same statistics. (ii) The statistics of $\ln \left(\epsilon_{f}\right)$ are non-Gaussian, which is shown by the visible asymmetry of the measured $H\left[\ln \left(\epsilon_{f}\right)\right]$ for large values of $|k|(\gtrsim 1.5)$. While the whole curve is non-Gaussian, part of the measured $H\left[\ln \left(\epsilon_{f}\right)\right]$ with $k \gtrsim-1.5$ can be fit to a Gaussian function, $H(k) / H_{0}=\exp \left[-\left(k-\mu^{\prime}\right)^{2} / 2 \sigma_{l}^{\prime 2}\right]$, with $\mu^{\prime}$ $=0.1$ and $\sigma_{l}^{\prime}=0.92$ (solid line). Because of the asymmetry of the measured $H\left[\ln \left(\epsilon_{f}\right)\right]$ for $k \lesssim-1.5$, the fitted values of $\mu^{\prime}$ and $\sigma_{l}^{\prime}$ show small deviations from the expected values of $\mu^{\prime}=0$ and $\sigma_{l}^{\prime}=1$ for a perfectly Gaussian distribution.

The Gaussian statistics for $\ln \left(\epsilon_{f}\right)$ indicates a log-normal distribution for $\epsilon_{f}$, which describes well the large dissipation fluctuations with $\left[\ln \left(\epsilon_{f}\right)-\mu\right] / \sigma_{\ell} \gtrsim-1.5$. Log-normal distributions have been used to describe the intermittent nature of viscous dissipation fluctuations $[1,36]$. In fact, the cascade picture for the viscous dissipation field with rare localized regions of strong and/or weak energy dissipations may also apply to the thermal dissipation field in turbulent convection. Such localized regions are naturally identified as thermal plumes in turbulent convection, as evidenced by our finding that $\epsilon_{f}(\mathbf{r})$ is dominated by contributions from the plumes. It was also shown recently [37] that both the size and the "heat content" of the thermal plumes exhibit log-normal distributions. Log-Poisson distributions were also proposed to describe the intermittency of fluctuations of the viscous dissipation [38,39]. It is known that log-Poisson becomes similar to log-normal when its mean value is large. For small mean values, log-Poisson is asymmetric with a long tail for large fluctuations. Such a long tail distribution, however, is opposite to that shown in Fig. 13(b).

\section{Near the sidewall}

Figure 14 shows the time series data for the local temperature fluctuation $\delta T(t)$, temperature derivative $(d T / d z)(t)$, two components of the thermal dissipation rate $\epsilon_{f}^{x}(t)$ and $\boldsymbol{\epsilon}_{f}^{z}(t)$, and the total dissipation date $\boldsymbol{\epsilon}_{f}(t)$ (top to bottom curves). The measurements are made at position 2 shown in Fig. 1(b), which is in the mid-plane of the cell and at $1.5 \mathrm{~cm}$ away from the sidewall. This is the position at which the measured $\epsilon_{f}(x)$ reaches maximum (see Fig. 5). Compared to 


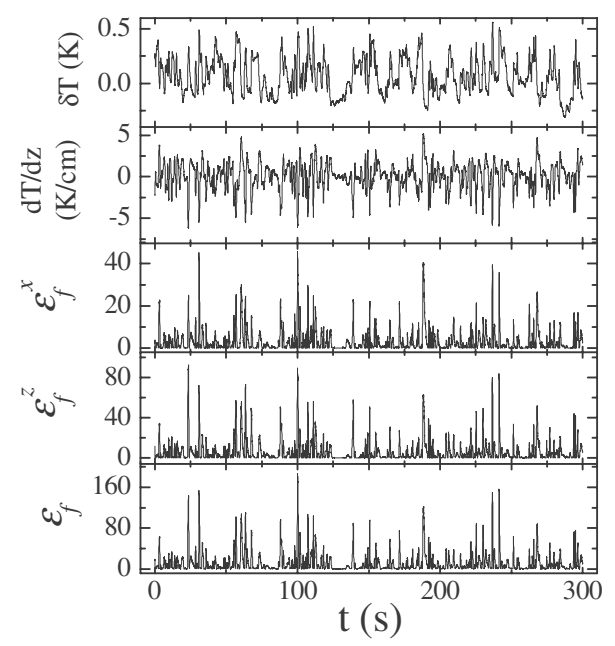

FIG. 14. Time series measurements of the local temperature fluctuation $\delta T(t)$, temperature derivative $(d T / d z)(t)$, two components of the thermal dissipation rate $\epsilon_{f}^{x}(t)$ and $\epsilon_{f}^{z}(t)$, and the total dissipation date $\epsilon_{f}(t)$ (top to bottom curves). All the measurements are made near the sidewall of the $\Gamma \simeq 1$ cell at $\mathrm{Ra}=3.9 \times 10^{9}$.

Fig. 11 (at the cell center), one finds more large temperature fluctuations near the sidewall, which are highly skewed toward one direction. These large upward going spikes are associated with the rising warm plumes in the region. It is seen that the thermal plumes do not arrive randomly, rather, they arrive in groups with a well-defined frequency $[34,35]$. The plume-generated temperature fluctuations give rise to large fluctuations in the temperature derivative and thermal dissipation rate, which are clearly shown in Fig. 14.

Figure 15 shows the normalized histograms $H(d T / d x) / H_{0}$ (diamonds), $H(d T / d y) / H_{0}$ (circles), and $H(d T / d z) / H_{0}$ (triangles) for the three components of the temperature gradient vector near the sidewall. Similar to the situation at the cell center, fluctuations of the two horizontal components are largely symmetric relative to the zero mean, and their histograms overlap well over an amplitude range of more than 5 decades. The functional form of the measured $H(d T / d x) / H_{0}$ [and $H(d T / d y) / H_{0}$ ], however, is different from that at the cell center with a sharp cusp near the origin. The measured

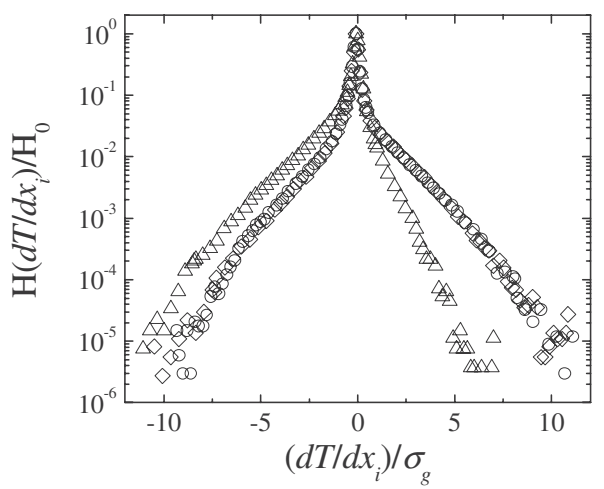

FIG. 15. Measured histograms $H(d T / d x) / H_{0}$ (diamonds), $H(d T / d y) / H_{0}$ (circles), and $H(d T / d z) / H_{0}$ (triangles) for the three components of the temperature gradient vector near the sidewall at $\mathrm{Ra}=3.96 \times 10^{9}$.
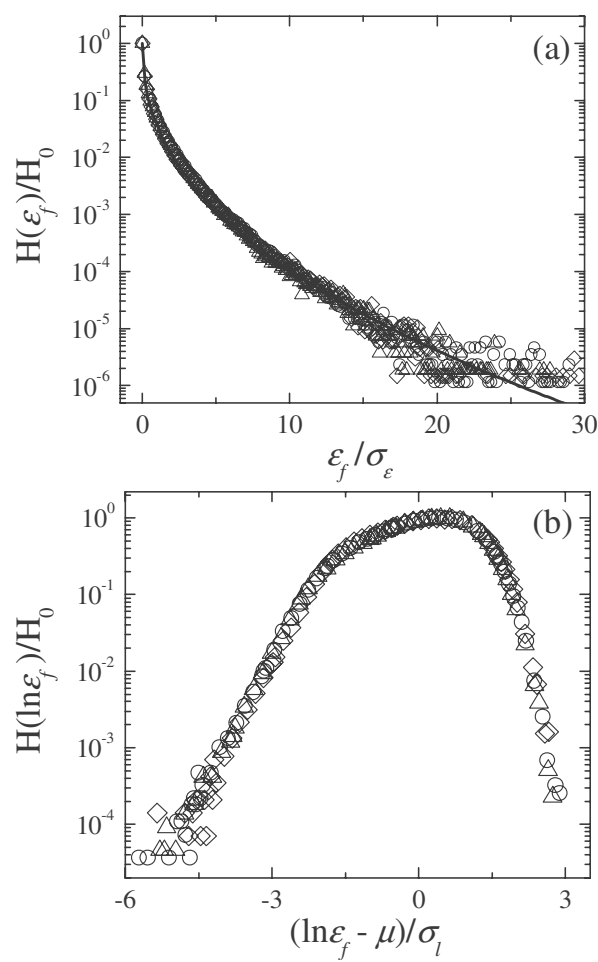

FIG. 16. (a) Measured histograms $H\left(\epsilon_{f}\right) / H_{0}$ as a function of $\epsilon_{f} / \sigma_{\epsilon}$. The measurements are made near the sidewall with three values of $\mathrm{Ra}: 1.7 \times 10^{9}$ (diamonds), $3.9 \times 10^{9}$ (circles), and 8.2 $\times 10^{9}$ (triangles). The solid line shows the fitted function, $H\left(\epsilon_{f}\right) / H_{0}=\exp \left[-c\left(\epsilon_{f} / \sigma_{\epsilon}\right)^{d}\right]$, with $c=3.32$ and $d=0.44$. (b) Histograms $H\left[\ln \left(\epsilon_{f}\right)\right] / H_{0}$ as a function of the reduced variable, $k$ $\equiv\left[\ln \left(\epsilon_{f}\right)-\mu\right] / \sigma_{l}$, using the same data in (a).

$H(d T / d z) / H_{0}$ near the sidewall shows a higher degree of asymmetry compared with that at the cell center (see Fig. 12) and is strongly skewed toward negative derivatives. The negative skewness is caused by the fact that there are many warm plumes in the region (see Fig. 14) and they lose heat while moving upward toward the top plate.

Figure 16(a) shows the normalized histograms $H\left(\epsilon_{f}\right) / H_{0}$ as a function of $\epsilon_{f} / \sigma_{\epsilon}$ near the sidewall. Similar to Fig. 13 (at the cell center), the measured histograms for different values of Ra can all be brought into coincidence, once $H\left(\epsilon_{f}\right)$ is normalized by its maximum value $H_{0}$ and $\epsilon_{f}$ is scaled by its rms value $\sigma_{\epsilon}$. The shape of the normalized histogram can be well described by a stretched exponential function, $H\left(\epsilon_{f}\right) / H_{0}=\exp \left[-c\left(\epsilon_{f} / \sigma_{\epsilon}\right)^{d}\right]$, over an amplitude range of almost 6 decades. The solid curve in Fig. 16(a) shows a fit with $c=3.32$ and $d=0.44$. The obtained value of $d$ near the sidewall is larger than that at the cell center. As mentioned earlier, there are more thermal plumes near the sidewall, which increases the rms value of $\epsilon_{f}$ and thus reduces the value of the normalized variable $\epsilon_{f} / \sigma_{\epsilon}$.

Figure 16(b) shows the normalized histograms $H\left[\ln \left(\epsilon_{f}\right)\right] / H_{0}$ of $\ln \left(\epsilon_{f}\right)$ as a function of the reduced variable, $k=\left[\ln \left(\epsilon_{f}\right)-\mu\right] / \sigma_{\ell}$, using the same data shown in Fig. 16(a). Compared to Fig. 13(b) (at the cell center), the measured $H\left[\ln \left(\epsilon_{f}\right)\right] / H_{0}$ near the sidewall shows a higher degree of asymmetry and is strongly skewed toward negative fluctuations. It has an exponential tail for large negative fluctuations 


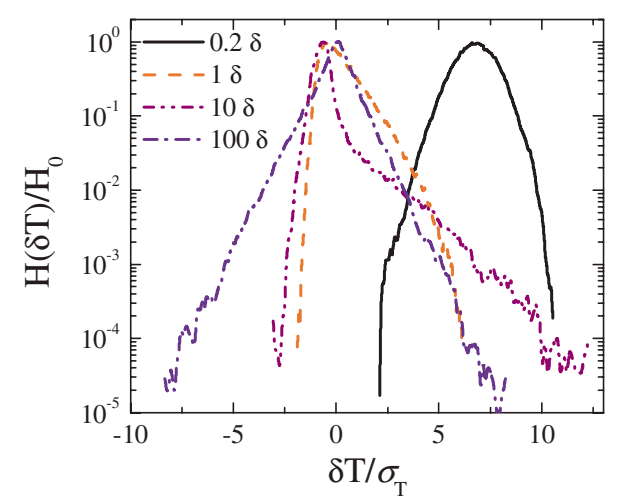

FIG. 17. (Color online) Measured temperature histograms $H(\delta T) / H_{0}$ as a function of $\delta T / \sigma_{T}$ along the central axis (the $z$ axis) of the $\Gamma \simeq 1$ cell. The measurements are made at $\mathrm{Ra}=1.7 \times 10^{9}$ with four different values of $z$ : $0.2 \delta$ (solid line), $1.0 \delta$ (dashed line), $10 \delta$ (dashed-dotted-dotted line), and $10^{2} \delta$ (dashed-dotted line). Here $\delta$ $(\simeq 1.0 \mathrm{~mm})$ is the boundary layer thickness at $\mathrm{Ra}=1.7 \times 10^{9}$.

in the range $\left[\ln \left(\epsilon_{f}\right)-\mu\right] / \sigma_{\ell} \lesssim-2$. (This corresponds to very small fluctuations in $\epsilon_{f}$.) We believe that the large deviations from the log-normal distribution are caused by the strong anisotropic flow generated by the thermal plumes near the sidewall. Because the plume-concentrated sidewall region has different statistical properties compared with the cell center, the statistical analysis of temperature fluctuations and their derivatives in the two regions should be carried out separately, instead of combining the two together [40].

\section{Near the lower conducting plate}

The fact that warm plumes are emitted from the thin thermal boundary layer near the bottom conducting plate makes this region particularly interesting for the study of changes of the statistical property of temperature fluctuations and their derivatives with the measuring position $z$. Figure 17 shows the measured temperature histograms $H(\delta T) / H_{0}$ as a function of $\delta T / \sigma_{T}$ along the central axis of the cell with five different values of $z$. Deep inside the thermal boundary layer $(z=0.2 \delta)$, temperature fluctuations are symmetric and the measured $H(\delta T) / H_{0}$ (solid line) is close to a Gaussian distribution. Because the bulk fluid temperature $T_{0}$ is used as a reference temperature, the peak value of the measured $H(\delta T) / H_{0}$ is shifted to the right-hand side with a mean value equal to the difference between the local mean and $T_{0}$. As $z$ is moved across the boundary layer thickness and to the region $\delta \lessgtr z \lesssim 10 \delta$, the measured $H(\delta T) / H_{0}$ (dashed and dashed-dotted-dotted lines) becomes more and more asymmetric. When $z$ is further moved to the central region, the degree of asymmetry of the measured $H(\delta T) / H_{0}$ reduces. At the cell center $(z \simeq 100 \delta), H(\delta T) / H_{0}$ becomes symmetric again and has a simple exponential form (dashed-dotted lines). This change of skewness for temperature fluctuations at different values of $z$ was also observed in recent numerical [40] and experimental [41] studies.

Figure 18(a) shows how the measured histograms $H(d T / d y)$ of the horizontal temperature derivative, $d T / d y$, change with the measuring position $z$. In the plot $H(d T / d y)$ is normalized by its maximum value $H_{0}$ and $d T / d y$ is scaled
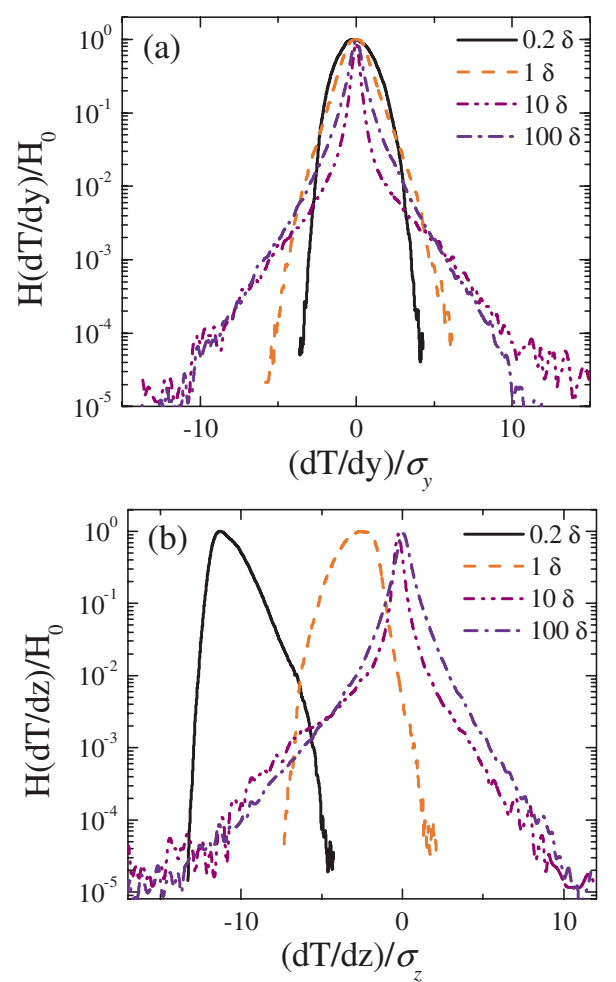

FIG. 18. (Color online) (a) Measured histograms $H(d T / d y) / H_{0}$ as a function of $(d T / d y) / \sigma_{y}$ along the central axis (the $z$ axis) of the $\Gamma \simeq 1$ cell. (b) Measured histograms $H(d T / d z) / H_{0}$ as a function of $(d T / d z) / \sigma_{z}$ along the $z$ axis. The measurements in (a) and (b) are made at $\mathrm{Ra}=1.7 \times 10^{9}$ with four different values of $z: 0.2 \delta$ (solid line), $1.0 \delta$ (dashed line), $10 \delta$ (dashed-dotted-dotted line), and $10^{2} \delta$ (dashed-dotted line). Here $\delta(\simeq 1.0 \mathrm{~mm})$ is the boundary layer thickness at $\mathrm{Ra}=1.7 \times 10^{9}$.

by its rms value $\sigma_{y}$. Unlike the local temperature, fluctuations of the horizontal temperature derivative are symmetric and have a zero mean for all values of $z$. The shape of $H(d T / d y)$ changes with $z$ with a long tail developed for large fluctuations of $d T / d y$ when $z$ is moved away from the boundary layer. If a stretched exponential function, $H(d T / d y) / H_{0}=\exp \left\{-c\left[(d T / d y) / \sigma_{\epsilon}\right]^{d}\right\}$, is used to describe the functional form, we find the value of the exponent $d$ decreases with increasing $z$ from a value close to 2 (Gaussian) for $z=0.2 \delta$ to a value smaller than 1 for $z \simeq 100 \delta$ (cell center). It is also found that the histograms $H(d T / d x)$ for the other horizontal temperature derivative, $d T / d x$, measured at different values of $z$, have the same shape as that of $H(d T / d y)$ (see, e.g., Fig. 15), indicating that fluctuations of the temperature derivatives are isotropic in the horizontal plane perpendicular to the gravity.

Figure 18(b) shows the measured histograms $H(d T / d z) / H_{0}$ of the vertical temperature derivative, $d T / d z$, as a function of $(d T / d z) / \sigma_{z}$ for five different values of $z$. Here $\sigma_{z}$ is the rms value of $d T / d z$. In contrast to the horizontal temperature derivatives, fluctuations of the vertical temperature derivative are asymmetric in the region $\delta \leq z$ $\lessgtr 10 \delta$ and have a nonzero mean whose absolute value decreases with increasing $z$. As mentioned earlier, the measured $d T / d z$ has a negative mean value, because the rising warm plumes near the lower conducting plate lose heat while mov- 

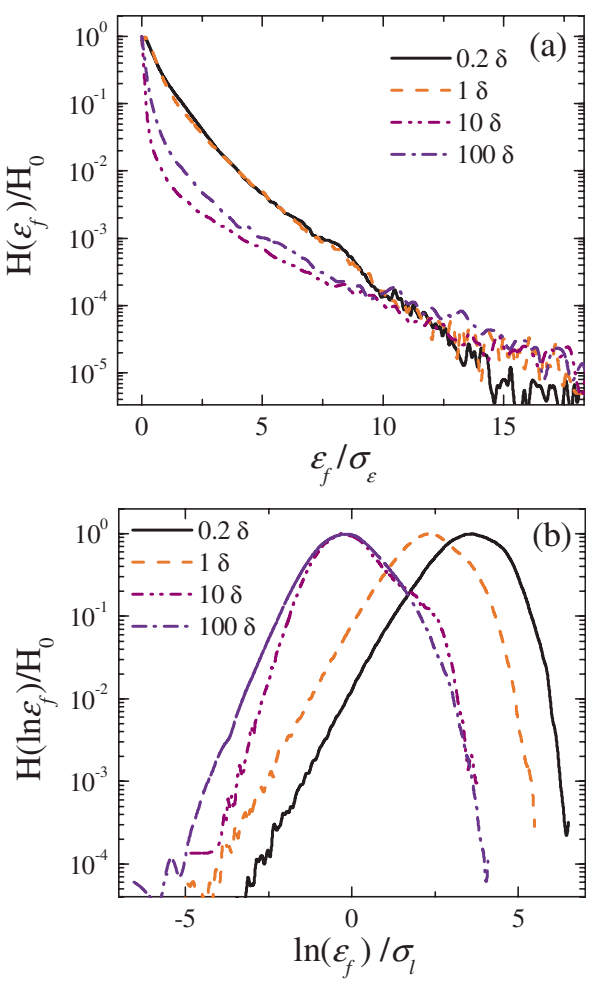

FIG. 19. (Color online) (a) Measured histograms $H\left(\epsilon_{f}\right) / H_{0}$ as a function of $\epsilon_{f} / \sigma_{\epsilon}$ along the $z$ axis of the $\Gamma \simeq 1$ cell. The measurements are made at $\mathrm{Ra}=1.7 \times 10^{9}$ with four different values of $z$ : $0.2 \delta$ (solid line), $1.0 \delta$ (dashed line), $10 \delta$ (dashed-dotted-dotted line), and $10^{2} \delta$ (dashed-dotted line). (b) Histograms $H\left[\ln \left(\epsilon_{f}\right)\right] / H_{0}$ as a function of the reduced variable, $\ln \left(\epsilon_{f}\right) / \sigma_{l}$, using the same data in (a).

ing upward. At the cell center $(z \simeq 100 \delta), H(d T / d z) / H_{0}$ becomes approximately symmetric and has a shape similar to that for the horizontal temperature derivatives (see Fig. 12).

The evolution of the measured histograms $H\left(\epsilon_{f}\right) / H_{0}$ for the local thermal dissipation rate $\epsilon_{f}$ along the $z$ axis is shown in Fig. 19(a). In the plot $H\left(\epsilon_{f}\right)$ is normalized by its maximum value $H_{0}$ and $\epsilon_{f}$ is scaled by its rms value $\sigma_{\epsilon}$. As $z$ is moved away from the boundary layer to the cell center, the measured $H\left(\epsilon_{f}\right) / H_{0}$ develops a long tail for large fluctuations of $\epsilon_{f}$. This long tail has a stretched exponential-like shape and becomes more extended with increasing $z$. Similar behavior is also observed in the measured $H\left(\epsilon_{f}\right) / H_{0}$ with increasing $\mathrm{Ra}$ (at a fixed value of $z$ ) (not shown). The extended long tail in the measured $H\left(\epsilon_{f}\right) / H_{0}$ indicates an increasing degree of small-scale intermittency of the thermal dissipation field [40].

Figure 19(b) shows how the histogram $H\left[\ln \left(\epsilon_{f}\right)\right] / H_{0}$ of $\ln \left(\epsilon_{f}\right)$ changes with the measuring position $z$. In the plot a reduced variable, $\ln \left(\epsilon_{f}\right) / \sigma_{l}$, is used for the horizontal axis with $\sigma_{l}$ being the rms value of $\ln \left(\epsilon_{f}\right)$. Similar to Fig. 16(b) (near the sidewall), the measured $H\left[\ln \left(\epsilon_{f}\right)\right] / H_{0}$ in the plumeconcentrated region $(z \lesssim 10 \delta)$ shows a higher degree of asymmetry. It has an exponential-like tail for large negative fluctuations. As $z$ is moved toward the cell center, the flow field becomes more isotropic and the resulting $H\left[\ln \left(\epsilon_{f}\right)\right] / H_{0}$ is more symmetric, having a shape close to a log-normal distribution for $\epsilon_{f}$ (see Fig. 13).

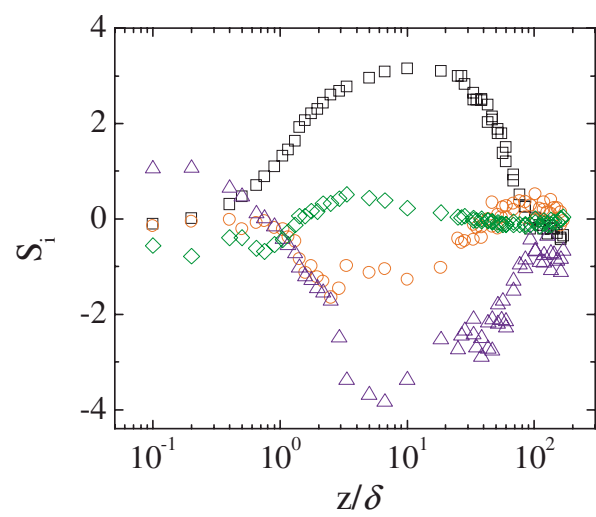

FIG. 20. (Color online) Measured skewness of the local temperature fluctuation $\delta T(t)$ (squares), horizontal temperature derivative $(d T / d y)(t)$ (circles), vertical temperature derivative $(d T / d z)(t)$ (triangles), and logarithmic dissipation rate $\ln \left(\epsilon_{f}\right)(t)$ (diamonds) as a function of the normalized distance $z / \delta$ along the central axis of the $\Gamma \simeq 1$ cell. All the measurements are made at $\mathrm{Ra} \simeq 6.0 \times 10^{9}$.

To quantitatively characterize the change of symmetry of the measured distribution functions, we calculate the skewness of a fluctuating variable $\theta_{i}$, defined as

$$
S_{i}=\frac{\left\langle\theta_{i}^{3}\right\rangle}{\left\langle\theta_{i}^{2}\right\rangle^{3 / 2}},
$$

where $\theta_{i}$ denotes $\delta T, d T / d y, d T / d z$, or $\ln \left(\epsilon_{f}\right)$. For symmetric distributions (in isotropic flows), one has $S_{i}=0$. Figure 20 shows the measured skewness $S_{i}$ of the local temperature fluctuation $\delta T$ (squares), horizontal temperature derivative $d T / d y$ (circles), vertical temperature derivative $d T / d z$ (triangles), and logarithmic dissipation rate $\ln \left(\epsilon_{f}\right)$ (diamonds) as a function of the normalized distance $z / \delta$ along the $z$ axis. It is seen that the flow field along the $z$ axis can be divided into three distinctive regions. (i) In the central core region $(z$ $\gtrsim 60 \delta$ ), the flow field is nearly isotropic and all the distribution functions are symmetric with skewness $S_{i}$ close to zero. (ii) Inside the thermal boundary layer $(z \lesssim \delta)$, the local temperature fluctuation $\delta T$ and the horizontal temperature derivative $d T / d y$ are not affected by the vertical mean temperature gradient across the boundary layer. Therefore, their distribution functions are symmetric with $S_{i} \simeq 0$. Fluctuations of the vertical temperature derivative $d T / d z$ and the resulting $\ln \left(\epsilon_{f}\right)$, however, are coupled to the vertical mean temperature gradient in the region, giving rise to a small value of skewness for each case with opposite signs. (iii) In the plumedominated mixing zone $(\delta \leqq z \leqq 60 \delta)$, the flow field is highly anisotropic and all the distribution functions are asymmetric with a peak value of $S_{i}$ located at $z \sim 10 \delta$. Similar changes in skewness were also found in a recent numerical study [40].

\section{SUMMARY}

We have systematically studied the thermal dissipation field and its statistical properties in turbulent RayleighBénard convection. A local temperature gradient probe consisting of four identical thermistors is made to measure the normalized thermal dissipation rate $\epsilon_{N}(\mathbf{r}, t)$ in two convec- 
tion cells filled with water. The aspect ratio of the two upright cylindrical cells used is $\Gamma \simeq 1$ and $\Gamma \simeq 0.5$, respectively. The measurements were conducted at a fixed Prandtl number $(\operatorname{Pr} \simeq 5.5)$ and over varying Rayleigh numbers $\left(8.9 \times 10^{8}\right.$ $\lesssim \operatorname{Ra} \lesssim 9.3 \times 10^{9}$ ) and spatial positions $\mathbf{r}$ across the entire convection cell. From the spatial measurements of the timeaveraged thermal dissipation rate, $\epsilon_{N}(\mathbf{r}) \equiv\left\langle\epsilon_{N}(\mathbf{r}, t)\right\rangle_{t}$, we obtain the following physical picture about the thermal dissipation field in turbulent convection.

First, the measured $\epsilon_{N}(\mathbf{r})$ can be decomposed into two contributions, $\epsilon_{N}(\mathbf{r})=\epsilon_{f}(\mathbf{r})+\epsilon_{m}(\mathbf{r})$, with $\epsilon_{m}(\mathbf{r})$ resulting from the mean temperature gradient and $\epsilon_{f}(\mathbf{r})$ coming from the fluctuations. In the bulk region outside the thermal boundary layers, $\epsilon_{f}(\mathbf{r})$ is the dominant contribution to $\epsilon_{N}(\mathbf{r})$. It is generated by the detached thermal plumes and occupies mainly in the plume-dominated region near the sidewall and near the boundary layers $(z / \delta>1)$. In the central region of the cell, the measured $\epsilon_{f}(\mathbf{r})$ has a minimal value because of the small number of thermal plumes present in the region. Inside the thermal boundary layers $(z / \delta \lesssim 1), \epsilon_{m}(\mathbf{r})$ is the dominant contribution to $\epsilon_{N}(\mathbf{r})$. It comes from the mean temperature gradient in the direction perpendicular to the conducting surfaces. This spatial decomposition of $\epsilon_{N}(\mathbf{r})=\epsilon_{f}(\mathbf{r})+\epsilon_{m}(\mathbf{r})$ is consistent with the first scenario of the GL theory [7].

Second, while the spatial decomposition, $\epsilon_{N}(\mathbf{r})=\epsilon_{f}(\mathbf{r})$ $+\epsilon_{m}(\mathbf{r})$, is consistent with the first scenario of the GL theory [7], the measured Ra dependence of $\epsilon_{f}(\mathbf{r})$ and $\epsilon_{m}(\mathbf{r})$ does not agree with this scenario. It is found that $\epsilon_{f}(\mathbf{r})$ throughout the bulk region scales as $\mathrm{Ra}^{-0.33}$, whereas $\epsilon_{m}(\mathbf{r})$ inside the thermal boundary layers scales with $\mathrm{Ra}$ differently $\left[\boldsymbol{\epsilon}_{m}(\mathbf{r})\right.$ $\left.\sim \mathrm{Ra}^{0.63}\right]$. The measurements clearly reveal two competing effects of turbulence. On the one hand, temperature fluctuations are destroyed in the bulk region and their contribution to $\epsilon_{N}(\mathbf{r})$ decreases with increasing Ra. On the other hand, the temperature gradient (and hence the thermal dissipation) is enhanced near the upper and lower conducting plates, be- cause of the thinning of the boundary layers with increasing Ra. With these two competing effects, we conclude that the thermal plumes and boundary layers are two different kinds of dynamic structures and cannot be treated equally, as was suggested by the second scenario of the GL theory $[7,10]$.

Finally, we studied the statistical properties of the temperature derivatives $\left(d T / d x_{i}\right)(t)\left(x_{i}=x, y, z\right)$ and the resulting dissipation rate $\epsilon_{f}(\mathbf{r}, t)$ at three representative locations in the convection cell: At the center, near the sidewall and near the lower conducting plate. We measured the probability density functions (histograms) of $\left(d T / d x_{i}\right)(t)$ and $\epsilon_{f}(\mathbf{r}, t)$ with varying $\mathrm{Ra}$. It is found that the measured histograms are invariant with $\mathrm{Ra}$, once the statistical variables concerned are normalized by their rms values. The histogram of the local thermal dissipation rate is characterized both in terms of $\epsilon_{f}$ and $\ln \left(\epsilon_{f}\right)$. By carefully examining the evolution of the measured histograms of $\delta T, d T / d y, d T / d z$, and $\ln \left(\epsilon_{f}\right)$, we find three different statistical behaviors along the central axis of the cell (the $z$ axis). (i) In the central core region $(z \gtrsim 60 \delta)$, the flow field is nearly isotropic and all the histograms are symmetric with skewness $S_{i}$ close to zero. (ii) Inside the thermal boundary layer $(z \lesssim \delta)$, the measured histograms of $\delta T$ and $d T / d y$ are not affected by the vertical mean temperature gradient across the boundary layer and remain symmetric with $S_{i} \simeq 0$. However, fluctuations of $d T / d z$ and the resulting $\ln \left(\epsilon_{f}\right)$ are coupled to the vertical mean temperature gradient in the region. (iii) In the plume-dominated mixing zone $(\delta$ $\lesssim z \leqq 60 \delta$ ), the flow field is highly anisotropic and all the measured histograms are asymmetric with a peak value of $S_{i}$ located at $z \sim 10 \delta$.

\section{ACKNOWLEDGMENTS}

We have benefited from illuminating discussions with K.-Q. Xia, D. Lohse, and E. Ching. This work was supported by the Hong Kong Research Grants Council under Grant No. HKUST-602907.
[1] U. Frisch, Turbulence: The Legacy of A. N. Kolmogorov (Cambridge University Press, Cambridge, UK, 1995).

[2] B. W. Zeff, D. D. Lanterman, R. McAllister, R. Roy, E. J. Kostelich, and D. P. Lathrop, Nature (London) 421, 146 (2003).

[3] K. R. Sreenivasan, R. A. Antonia, and H. Q. Danh, Phys. Fluids 20, 1238 (1977).

[4] R. R. Prasad, C. Meneveau, and K. R. Sreenivasan, Phys. Rev. Lett. 61, 74 (1988).

[5] D. Kushnir, J. Schumacher, and A. Brandt, Phys. Rev. Lett. 97, 124502 (2006).

[6] E. Siggia, Annu. Rev. Fluid Mech. 26, 137 (1994).

[7] S. Grossmann and D. Lohse, J. Fluid Mech. 407, 27 (2000).

[8] S. Grossmann and D. Lohse, Phys. Rev. Lett. 86, 3316 (2001).

[9] S. Grossmann and D. Lohse, Phys. Rev. E 66, 016305 (2002).

[10] S. Grossmann and D. Lohse, Phys. Fluids 16, 4462 (2004).

[11] B. Castaing, G. Gunaratne, F. Heslot, L. Kadanoff, A. Libchaber, S. Thomae, X.-Z. Wu, S. Zaleski, and G. Zanetti, J. Fluid Mech. 204, 1 (1989).
[12] J. A. Glazier, T. Segawa, A. Naert, and M. Sano, Nature (London) 398, 307 (1999).

[13] X. Chavanne, F. Chillà, B. Chabaud, B. Castaing, and B. Hébral, Phys. Fluids 13, 1300 (2001).

[14] J. J. Niemela, L. Skrbek, K. R. Sreenivasan, and R. J. Donnelly, Nature (London) 404, 837 (2000).

[15] J. J. Niemela and K. R. Sreenivasan, J. Fluid Mech. 557, 411 (2006).

[16] G. Ahlers and X.-C. Xu, Phys. Rev. Lett. 86, 3320 (2001).

[17] D. Funfschilling, E. Brown, A. Nikolaenko, and G. Ahlers, J. Fluid Mech. 536, 145 (2005).

[18] K.-Q. Xia, S. Lam, and S.-Q. Zhou, Phys. Rev. Lett. 88, 064501 (2002)

[19] C. Sun, L.-Y. Ren, H. Song, and K.-Q. Xia, J. Fluid Mech. 542, 165 (2005)

[20] Y.-B. Du and P. Tong, J. Fluid Mech. 407, 57 (2000).

[21] X.-L. Qiu, X.-D. Shang, P. Tong, and K.-Q. Xia, Phys. Fluids 16, 412 (2004).

[22] X.-D. Shang, X.-L. Qiu, P. Tong, and K.-Q. Xia, Phys. Rev. E 
70, 026308 (2004).

[23] G. Ahlers, E. Brown, and A. Nikolaenko, J. Fluid Mech. 557, 347 (2006).

[24] Y.-B. Du and P. Tong, Phys. Rev. E 63, 046303 (2001).

[25] X.-Z. He, P. Tong, and K.-Q. Xia, Phys. Rev. Lett. 98, 144501 (2007).

[26] S.-L. Lui and K.-Q. Xia, Phys. Rev. E 57, 5494 (1998).

[27] X.-L. Qiu and P. Tong, Phys. Rev. E 64, 036304 (2001).

[28] C. Sun, K.-Q. Xia, and P. Tong, Phys. Rev. E 72, 026302 (2005).

[29] X.-D. Shang, X.-L. Qiu, P. Tong, and K.-Q. Xia, Phys. Rev. Lett. 90, 074501 (2003).

[30] R. Verzicco and R. Camussi, J. Fluid Mech. 477, 19 (2003).

[31] R. Verzicco, Eur. Phys. J. B 35, 133 (2003).
[32] H.-D. Xi, S. Lam, and K.-Q. Xia, J. Fluid Mech. 503, 47 (2004).

[33] E. S. C. Ching, H. Guo, X.-D. Shang, P. Tong, and K.-Q. Xia, Phys. Rev. Lett. 93, 124501 (2004).

[34] X.-L. Qiu and P. Tong, Phys. Rev. Lett. 87, 094501 (2001).

[35] X.-L. Qiu and P. Tong, Phys. Rev. E 66, 026308 (2002).

[36] A. S. Gurvich and A. M. Yaglom, Phys. Fluids 10, S59 (1967).

[37] S.-Q. Zhou and K.-Q. Xia, Phys. Rev. Lett. 89, 184502 (2002).

[38] B. Dubrulle, Phys. Rev. Lett. 73, 959 (1994).

[39] Z.-S. She and E. C. Waymire, Phys. Rev. Lett. 74, 262 (1995).

[40] M. S. Emran and J. Schumacher, J. Fluid Mech. 611, 13 (2008).

[41] A. Belmonte, A. Tilgner, and A. Libchaber, Phys. Rev. E 50, 269 (1994). 